\title{
Dynamical exchange-correlation potentials for the electron liquid in the spin channel
}

\author{
Zhixin Qian and Giovanni Vignale \\ Department of Physics, University of Missouri, Columbia, Missouri 65211, USA
}

(Received 17 June 2003; published 20 November 2003)

\begin{abstract}
The components of the exchange-correlation kernel tensor of an isotropic electron liquid in the spin channel have the structure $f_{\mathrm{xc},-}^{L, T}(q, \omega) \stackrel{q \rightarrow 0}{\rightarrow} A(\omega) / q^{2}+B^{L, T}(\omega)$, where $L$ denotes the longitudinal component and $T$ the transverse component relative to the direction of the wave vector $\mathbf{q}$. In this paper we calculate analytically the high- and low-frequency limits of $A(\omega)$ and $B^{L, T}(\omega)$ and combine these limiting forms with the KramersKrönig dispersion relations to obtain approximations for $A(\omega)$ and $B^{L, T}(\omega)$ at all frequencies.
\end{abstract}

DOI: 10.1103/PhysRevB.68.195113

PACS number(s): 71.45.Gm, 71.15.Mb, 71.10.-w

\section{INTRODUCTION}

The spin-resolved local field factor $G_{\sigma \sigma^{\prime}}(q, \omega)$ has often been used to describe exchange-correlation (xc) effects in the electron liquid. ${ }^{1,2}$ These effects arise from the antisymmetry of the many-body wave function (exchange) and from the Coulomb repulsion between the electrons (correlation). The equivalent concept of exchange-correlation kernel, defined as

$$
f_{\mathrm{xc}, \sigma \sigma^{\prime}}(q, \omega)=-v(q) G_{\sigma \sigma^{\prime}}(q, \omega),
$$

where $v(q)$ is the Fourier transform of the Coulomb potential, is more frequently used in time dependent (spin) density functional theory. ${ }^{3-6} f_{\mathrm{xc}, \sigma \sigma^{\prime}}(q, \omega)$ determines the xc potential $V_{\mathrm{xc}, \sigma}(q, \omega)$ created by a small density fluctuation $\delta n_{\sigma^{\prime}}(q, \omega)$ according to the formula

$$
V_{\mathrm{xc}, \sigma}(q, \omega)=\sum_{\sigma^{\prime}} f_{\mathrm{xc}, \sigma \sigma^{\prime}}(q, \omega) \delta n_{\sigma^{\prime}}(q, \omega) .
$$

It is customary in density functional theory to replace the nonlocal relation (2) by the local density approximation

$$
V_{\mathrm{xc}, \sigma}(\mathbf{r}, \omega) \simeq \sum_{\sigma^{\prime}} f_{\mathrm{xc}, \sigma \sigma^{\prime}}(q=0, \omega) \delta n_{\sigma^{\prime}}(\mathbf{r}, \omega),
$$

where the $q=0$ kernel $f_{\mathrm{xc}, \sigma \sigma^{\prime}}(q=0, \omega)$ is evaluated at the local equilibrium density. Unfortunately, this cannot be done for the spin-resolved $f_{\mathrm{xc}, \sigma \sigma^{\prime}}(q, \omega)$, for the simple reason that the $q \rightarrow 0$ limit of this function does not exist. In fact, one has $^{7}$

$$
f_{\mathrm{xc}, \sigma \sigma^{\prime}}(q, \omega) \simeq \frac{A(\omega)}{q^{2}} \frac{\sigma \sigma^{\prime} n^{2}}{4 n_{\sigma} n_{\sigma^{\prime}}}+B_{\sigma \sigma^{\prime}}(\omega)+O\left(q^{2}\right),
$$

where $n_{\sigma}$ is the density of $\sigma$-spin electrons $(\sigma=+1$ for $\uparrow$-spin and $\sigma=-1$ for $\downarrow$-spin), and $n=n_{\uparrow}+n_{\downarrow}$ is the total density. $A(\omega)$ and $B_{\sigma \sigma^{\prime}}(\omega)$ are finite functions of frequency. The small- $q$ divergence cancels out in the density channel, i.e., when one sums over the spin indices, but not in any other combination of the matrix elements $f_{\mathrm{xc}, \sigma \sigma^{\prime}}(q, \omega)$.

It has recently been pointed out, ${ }^{7}$ that this difficulty can be avoided by switching to a more complete description in which the basic variables are the longitudinal and transverse components of the spin-resolved current density. In the spin- current density functional theory the xc potential is a vector potential $\mathbf{A}_{\mathrm{xc}, \sigma}$, which is related to the spin-resolved current density by the following relation:

$$
A_{\mathrm{xc}, \sigma}^{i}(\mathbf{q}, \omega)=\frac{q^{2}}{\omega^{2}} \sum_{j, \sigma^{\prime}} f_{\mathrm{xc}, \sigma \sigma^{\prime}}^{i j}(\mathbf{q}, \omega) \delta j_{\sigma^{\prime}}^{j}(\mathbf{q}, \omega),
$$

where $i$ and $j$ are Cartesian indices. In isotropic electron liquid, the tensor kernel $f_{\mathrm{xc}, \sigma \sigma^{\prime}}^{i j}(\mathbf{q}, \omega)$ can be described by its longitudinal $(L)$ and transverse $(T)$ components $f_{\mathrm{xc}, \sigma \sigma^{\prime}}^{L, T}(q, \omega)$, defined by the relation

$$
A_{\mathrm{xc}, \sigma}^{L, T}(q, \omega)=\frac{q^{2}}{\omega^{2}} \sum_{\sigma^{\prime}} f_{\mathrm{xc}, \sigma \sigma^{\prime}}^{L, T}(q, \omega) \delta j_{\sigma^{\prime}}^{L, T}(q, \omega),
$$

where $L$ and $T$ denote the longitudinal and transverse component relative to the direction of $\mathbf{q}$.

It is easy to verify that $f_{\mathrm{xc}, \sigma \sigma^{\prime}}^{L}(q, \omega)$ coincides with the usual $f_{\mathrm{xc}, \sigma \sigma^{\prime}}(q, \omega)$ of the standard density functional formalism, but $f_{\mathrm{xc}, \sigma \sigma^{\prime}}^{T}(q, \omega)$ is new. Both $f_{\mathrm{xc}, \sigma \sigma^{\prime}}^{L}(q, \omega)$ and $f_{\mathrm{xc}, \sigma \sigma^{\prime}}^{T}(q, \omega)$ have a small- $q$ expansion of the form (4),

$$
f_{\mathrm{xc}, \sigma \sigma^{\prime}}^{L, T}(q, \omega) \stackrel{q \rightarrow 0}{\rightarrow} \frac{A(\omega)}{q^{2}} \frac{\sigma \sigma^{\prime} n^{2}}{4 n_{\sigma} n_{\sigma^{\prime}}}+B_{\sigma \sigma^{\prime}}^{L, T}(\omega)+O\left(q^{2}\right),
$$

and for this reason the connection (6) between $A_{\mathrm{xc}, \sigma}^{L, T}(q, \omega)$ and $\delta j_{\sigma}^{L, T}(q, \omega)$ is well-behaved in the small- $q$ limit, i.e., local, as we wanted.

Against this background, the importance of having reliable expressions for $A(\omega)$ and $B_{\sigma \sigma^{\prime}}^{L, T}(\omega)$ is self-evident. These functions are also needed for a consistent description of effective interaction, including exchange of spin fluctuations. ${ }^{8-11}$

It is convenient to consider separately the density-density and the spin-spin "channels." In the density-density channel the small-q singularity cancels out and one is left with

$$
f_{\mathrm{xc}}^{L, T}(\omega)=\sum_{\sigma \sigma^{\prime}} B_{\sigma \sigma^{\prime}}^{L, T}(\omega) \frac{n_{\sigma^{\prime}} n_{\sigma^{\prime}}}{n^{2}} .
$$

Approximate expressions for $f_{\mathrm{xc}}^{L, T}(\omega)$ have been obtained by the present authors ${ }^{12}$ and, earlier, by several others. ${ }^{10,13,14}$ These formulas are extremely useful in time-dependent 
current-density functional theory, ${ }^{15-17}$ which has recently found interesting applications to the calculations of the optical spectra of solid ${ }^{18}$ and the polarizability of long polymer chains. ${ }^{19}$

In this paper we address the remaining part of the problem, namely, the calculation of the spin-spin channel quantities $A(\omega)$ and

$$
B^{L, T}(\omega)=\sum_{\sigma \sigma^{\prime}} B_{\sigma \sigma^{\prime}}^{L, T}(\omega) \sigma \sigma^{\prime} \frac{n_{\sigma} n_{\sigma^{\prime}}}{n^{2}}
$$

Concerning $A(\omega)$, we mainly build on the work briefly reported in Ref. 7: we supply detailed derivations of the results presented there without proof, and derive an exact relationship between the low-frequency behavior of $\operatorname{Re} A(\omega)$ and the Landau parameters. We also evaluate the low frequency behavior of $\operatorname{Im} A(\omega)$ within the mode-decoupling approximation, ${ }^{13,14}$ which is supposed to be exact in the high-density limit. The main part of this paper, however, is devoted to the study of $B^{L, T}(\omega)$.

First of all, we derive the high and low frequency limits of $\operatorname{Im} B^{L, T}(\omega)$. To this end, we express the small- $q$ limit of $\operatorname{Im} f_{\mathrm{xc}, \sigma \sigma^{\prime}}^{i j}(\mathbf{q}, \omega)$ in terms of four-point response functions, which are then treated perturbatively. The expansion is accurate to second order in the Coulomb interaction, yet we believe that it yields the exact asymptotic behavior at high frequency because in that limit the four-point response function of the interacting system reduces to that of the noninteracting one. In this way $\operatorname{Im} B^{L, T}(\omega)$ is found to decrease as $\left(1 / \omega^{3 / 2}\right)$, which is the same behavior that was previously established for $\operatorname{Im} f_{\mathrm{xc}}^{L, T}(\omega)$ in the density-density channel. ${ }^{13,14,20}$

The same small wavevector expansion is applied to the calculation of the low frequency behavior of $\operatorname{Im} B^{L, T}(\omega)$, but, in this case, the results are only valid in the high-density limit. We find that, in this limit, the difference between $\operatorname{Im} B^{L, T}(\omega)$ and $\operatorname{Im} f_{\mathrm{xc}}^{L, T}(\omega)$, vanishes to first order in $\omega$. We also show that $\operatorname{Im} B^{T}(\omega)=(3 / 4) \operatorname{Im} B^{L}(\omega)$ at small $\omega$. Furthermore, we establish a relation between $\operatorname{Re} B^{L, T}(0)$ and the Landau parameters parallel to the one obtained by Conti and Vignale $^{21}$ in the density-density channel.

We then construct approximate interpolation formulas for $\operatorname{Im} B^{L, T}(\omega)$ based on the above results. The philosophy is the same as that in Ref. 12. Basically, we attempt to improve the Gross-Kohn interpolation formula ${ }^{22,23}$ by introducing a peak at intermediate frequency. The position of the peak at about $\omega_{m}=3 \omega_{\mathrm{pl}} / 2$ (where $\omega_{\mathrm{pl}}$ is the plasmon frequency) is suggested by the mode-decoupling theory, while the strength of the peak is fixed (via dispersion relations) by the lowfrequency behavior of the real part of $B^{L, T}(\omega)$.

It turns out that the same type of interpolation for $\operatorname{Im} A(\omega)$ does not work well at "metallic densities." Therefore, we resort to the mode-decoupling approximation (already discussed in Ref. 7) to describe this function. The results obtained in this paper should be useful in current implementations of the spin-current-density functional theory, which aim at calculating the energy of spindependent excitations in complex electronic systems.
The paper is organized as follows. In Sec. II, we summarize the exact properties of $A(\omega)$ and $B^{L, T}(\omega)$. In Sec. III, we present the derivations of these exact properties. In Sec. IV, we present our interpolation formulas for $B^{L, T}(\omega)$ in paramagnetic state. Further technical details are presented in the two Appendixes.

\section{EXACT PROPERTIES}

We begin by listing, for ease of reference, the exact properties of $A(\omega)$ and $B^{L, T}(\omega)$. The derivations are very technical and will be presented in the next section.

First of all, we note that both $A(\omega)$ and $B^{L, T}(\omega)$ satisfy the Kramers-Krönig (KK) relations. For example, we have

$$
\operatorname{Re} A(\omega)-A(\infty)=P \int_{-\infty}^{\infty} \frac{d \omega^{\prime}}{\pi} \frac{\operatorname{Im} A\left(\omega^{\prime}\right)}{\omega^{\prime}-\omega},
$$

where $P$ is the principal part, and similarly for $B^{L, T}(\omega)$.

\section{A. Exact properties for $A(\omega)$}

The high-frequency behavior of $\operatorname{Im} A(\omega)$ is ${ }^{7}$

$$
\operatorname{Im} A(\omega) \stackrel{\omega \rightarrow \infty}{\rightarrow}-\frac{16 \pi e^{2}}{3} \frac{n_{\uparrow} n_{\downarrow}}{n^{2}} \frac{m e^{2}}{\sqrt{m \omega}} .
$$

The infinite frequency limit of $\operatorname{Re} A(\omega)$ is given by the third-moment sum rule r $^{24,25}$

$$
\operatorname{Re} A(\infty)=\lim _{q \rightarrow 0}\left(\frac{m}{n q}\right)^{2} M_{3}(q)=-\frac{16 \pi e^{2}}{3} \frac{n_{\uparrow} n_{\downarrow}}{n^{2}}\left[g_{\uparrow \downarrow}(0)-1\right],
$$

where $M_{3}(q)=-(1 / \pi V) \int_{-\infty}^{\infty} \omega^{3} \operatorname{Im} \chi_{s}(q, \omega) d \omega$ is the thirdmoment of the spin-spin response function, with $V$ the volume, and $g_{\uparrow \downarrow}(0)=g_{\uparrow \downarrow}(r=0)$, with $g_{\sigma \sigma^{\prime}}(r)$ the spinresolved pair correlation function.

In addition, $\operatorname{Re} A(\omega)$ and $\operatorname{Im} A(\omega)$ vanish at zero frequency as $\omega^{2}$ and $\omega^{3}$, respectively. It will be shown that

$$
\begin{gathered}
\lim _{\omega \rightarrow 0} A(\omega)=0, \\
1-\lim _{\omega \rightarrow 0} \frac{n A(\omega)}{m \omega^{2}}=\frac{1+F_{1}^{s} / 3}{1+F_{1}^{a} / 3},
\end{gathered}
$$

where $F_{l}^{s}$ and $F_{l}^{a}$ are spin-symmetric and spin-antisymmetric Landau parameters, respectively ${ }^{26}$ and

$$
\lim _{\omega \rightarrow 0} \frac{\operatorname{Im} A(\omega)}{\omega^{3}}=-\frac{m^{4} e^{4}}{18 \pi^{3} n^{2} k_{s}}\left[\frac{\pi}{2}-\frac{2 k_{F} k_{s}}{4 k_{F}^{2}+k_{s}^{2}}-\tan ^{-1} \frac{k_{s}}{2 k_{F}}\right],
$$

where $k_{s}$ is the screening wave vector $k_{s}=\sqrt{4 k_{F} / \pi a_{0}}, k_{F}$ the Fermi wave vector, and $a_{0}$ is the Bohr radius. The last two equations above are specialized to the paramagnetic case, and Eq. (15) is exact only in the high density limit. 


\section{B. Exact properties for $B^{L, T}(\omega)$}

The high-frequency behavior of $\operatorname{Im} B^{L, T}(\omega)$ is

$$
\operatorname{Im} B^{L, T}(\omega) \stackrel{\omega \rightarrow \infty}{\rightarrow}-c^{L, T} \frac{\pi m e^{4}}{(m \omega)^{3 / 2}},
$$

where $c^{L}=41 / 30, c^{T}=17 / 30$.

The infinite frequency limit of $B^{L, T}(\omega)$ is

$$
B^{L, T}(\infty)=\alpha^{L, T} \frac{t_{c}}{n}+\frac{\beta^{L, T}}{2} \int d \mathbf{r} v(\mathbf{r}) g_{-}(r),
$$

where $\alpha^{L}=2, \alpha^{T}=2 / 3, \beta^{L}=4 / 15, \beta^{T}=-2 / 15$, and $g_{-}(r)$ $=\Sigma_{\sigma \sigma^{\prime}}\left(n_{\sigma} n_{\sigma^{\prime}} / n^{2}\right) \sigma \sigma^{\prime} g_{\sigma \sigma^{\prime}}(r)$.

The zero-frequency limits of $B^{L, T}(\omega)$ are related to the Landau parameters as follows:

$$
B^{L}(0)=\frac{2 E_{F}}{n} \frac{4 F_{2}^{a} / 75+F_{0}^{a} / 3-F_{1}^{s} / 5}{1+F_{1}^{s} / 3}
$$

and

$$
B^{T}(0)=\frac{2 E_{F}}{n} \frac{F_{2}^{a} / 25-F_{1}^{s} / 15}{1+F_{1}^{s} / 3},
$$

where $E_{F}=k_{F}^{2} / 2 m$ is the Fermi energy. The low-frequency behavior of $\operatorname{Im} B^{L, T}(\omega)$ is determined by

$$
\lim _{\omega \rightarrow 0} \frac{\operatorname{Im} B^{L, T}(\omega)}{\omega}=-\left(\frac{m e^{2}}{n \pi}\right)^{2} k_{F} S^{L, T},
$$

where the dimensionless constants $S^{L, T}$ are given by

$$
\begin{aligned}
S^{L}= & -\frac{1}{45 \pi}\left\{5-(\lambda+5 / \lambda) \tan ^{-1} \lambda-\frac{2}{\lambda} \sin ^{-1} \frac{\lambda}{\sqrt{1+\lambda^{2}}}\right. \\
& \left.+\frac{2}{\lambda \sqrt{2+\lambda^{2}}}\left[\frac{\pi}{2}-\tan ^{-1} \frac{1}{\lambda \sqrt{2+\lambda^{2}}}\right]\right\}
\end{aligned}
$$

and

$$
S^{T}=\frac{3}{4} S^{L}
$$

In the above expression $\lambda \equiv 2 k_{F} / k_{s}$.

While Eq. (21) is perturbative, and therefore strictly valid only in the high density limit, the relation

$$
\lim _{\omega \rightarrow 0} \frac{\operatorname{Im} B^{T}(\omega)}{\omega}=\frac{3}{4} \lim _{\omega \rightarrow 0} \frac{\operatorname{Im} B^{L}(\omega)}{\omega}
$$

is nonperturbative and, as such, is expected to hold at all densities. The results listed in this subsection are specialized to the paramagnetic case, except for Eq. (17), which holds in general.

\section{DERIVATIONS}

\section{A. Small wave vector expansion for imaginary part of the $\mathrm{xc}$ kernel tensor $\operatorname{Im} f_{\mathrm{xc}, \sigma \sigma^{\prime}}^{i j}(\mathbf{q}, \boldsymbol{\omega})$}

To derive the exact results for $A(\omega)$ and $B^{L, T}(\omega)$ listed in Sec. II, we first establish the small- $q$ expansion of $\operatorname{Im} f_{\mathrm{xc}, \sigma \sigma^{\prime}}^{i j}(\mathbf{q}, \omega)$ in terms of four-point response functions. We begin with the exact expression

$$
\operatorname{Im} f_{\mathrm{xc}, \sigma \sigma^{\prime}}^{i j}(\mathbf{q}, \omega)=\frac{m^{2}}{V n_{\sigma} n_{\sigma^{\prime}}} \frac{\omega^{2}}{q^{2}} \operatorname{Im}\left\langle\left\langle j_{\sigma}^{i}(\mathbf{q}, t) ; j_{\sigma^{\prime}}^{j}(-\mathbf{q})\right\rangle\right\rangle_{\omega}
$$

where $\mathbf{j}_{\sigma}(\mathbf{q})$ is the spin-resolved current-density operator

$$
\mathbf{j}_{\sigma}(\mathbf{q})=\sum_{m=1}^{N} Q_{m \sigma} \mathbf{j}_{m}(\mathbf{q})
$$

where $Q_{m \sigma}=\left(1+\sigma \sigma_{m z}\right) / 2$ (with $\sigma_{m z}$ the $z$-component Pauli matrix for the $m$ th particle) picks the $\sigma$-spin component of the state of the $m$ th particle and

$$
\mathbf{j}_{m}(\mathbf{q}) \equiv \frac{1}{2 m}\left[\mathbf{P}_{m} \rho_{m}(\mathbf{q})+\rho_{m}(\mathbf{q}) \mathbf{P}_{m}\right]
$$

$\mathbf{P}_{m}$ and $\rho_{m}(\mathbf{q})$ are the momentum and density operators for the $m$ th particle.

The Zubarev product is defined as $\langle\langle A ; B\rangle\rangle_{\omega} \equiv$ $-i \int_{0}^{\infty} d t e^{i \omega t}\langle[A(t), B(0)]\rangle$. (The frequency argument will be omitted, for brevity, from now on.) Making use of this definition, one can show that Eq. (24) can be rewritten as

$$
\operatorname{Im} f_{\mathrm{xc}, \sigma \sigma^{\prime}}^{i j}(\mathbf{q}, \omega)=\frac{m^{2}}{V n_{\sigma} n_{\sigma^{\prime}}} \frac{1}{q^{2} \omega^{2}} \operatorname{Im}\left\langle\left\langle\ddot{j}_{\sigma}^{i}(\mathbf{q}, t) ; \ddot{j}_{\sigma^{\prime}}^{j}(-\mathbf{q})\right\rangle\right\rangle,
$$

where $\ddot{\mathbf{j}}_{\sigma}(\mathbf{q}, t)$ is the second time derivative of $\mathbf{j}_{\sigma}(\mathbf{q}, t)$. Operators without an explicit time argument are assumed to be evaluated at $t=0$. Since

$$
\dot{\mathbf{P}}_{m}=\frac{i}{V} \sum_{\mathbf{k}} \mathbf{k} \rho(-\mathbf{k}) \rho_{m}(\mathbf{k}) v(\mathbf{k}),
$$

commutes with the density fluctuations and

$$
\dot{\rho}_{m}(\mathbf{q})=-i \mathbf{q} \cdot \mathbf{j}_{m}(\mathbf{q})
$$

where $\rho(-\mathbf{k})=\sum_{m=1}^{N} \rho_{m}(\mathbf{k})$ and $N$ is the electron number, we see that

$$
\dot{\mathbf{j}}_{m}(\mathbf{q})=\frac{1}{2 m}\left[2 \dot{\mathbf{P}}_{m} \boldsymbol{\rho}_{m}(\mathbf{q})+\dot{\rho}_{m}(\mathbf{q}) \mathbf{P}_{m}+\mathbf{P}_{m} \dot{\boldsymbol{\rho}}_{m}(\mathbf{q})\right]
$$

and

$$
\begin{aligned}
\dddot{j}_{m}^{i}(\mathbf{q})= & \frac{1}{2 m}\left[\frac{2 i}{V} \sum_{\mathbf{k}} k_{i} v(\mathbf{k}) \frac{\partial}{\partial t}\left\{\rho(-\mathbf{k}) \rho_{m}(\mathbf{k}+\mathbf{q})\right\}\right. \\
& \left.-i \mathbf{q} \cdot\left\{\dot{P}_{m i}, \mathbf{j}_{m}(\mathbf{q})\right\}-i \mathbf{q} \cdot\left\{P_{m i}, \dot{\mathbf{j}}_{m}(\mathbf{q})\right\}\right]
\end{aligned}
$$


where $\{A, B\}=A B+B A$ is the anticommutator of $A$ and $B$. By using Eqs. (28), (29), and (30) in the above equation, we obtain

$$
\begin{aligned}
\dddot{j}_{m}^{i}(\mathbf{q})= & \frac{1}{2 m V} \sum_{\mathbf{k}} v(\mathbf{k}) \\
& \times\left[2 i k_{i} \frac{\partial}{\partial t}\left[\rho(-\mathbf{k}) \rho_{m}(\mathbf{k}+\mathbf{q})\right]\right. \\
& +k_{i}\left\{\rho(-\mathbf{k}) \rho_{m}(\mathbf{k}), \mathbf{q} \cdot \mathbf{j}_{m}(\mathbf{q})\right\} \\
& \left.+\frac{\mathbf{k} \cdot \mathbf{q}}{m}\left\{P_{m i}, \rho(-\mathbf{k}) \rho_{m}(\mathbf{k}+\mathbf{q})\right\}\right] \\
& -\frac{i}{2 m^{2} V}\left\{P_{m i},\left\{\mathbf{q} \cdot \mathbf{P}_{m}, \mathbf{q} \cdot \mathbf{j}_{m}(\mathbf{q})\right\}\right\} .
\end{aligned}
$$

Up to this point, the derivation is exact. The last term on the right-hand side (RHS) of Eq. (32) makes a contribution of higher order than $O\left(q^{0}\right)$ to $\operatorname{Im} f_{\mathrm{xc}, \sigma \sigma^{\prime}}^{i j}(\mathbf{q}, \omega)$, and is therefore dropped from now on.
Expanding the anticommutators in Eq. (32), one obtains

$$
\begin{aligned}
\ddot{j}_{m}^{i}(\mathbf{q})= & \frac{1}{m V} \sum_{\mathbf{k}} v(\mathbf{k})\left[i k_{i} \frac{\partial}{\partial t}\left[\rho(-\mathbf{k}) \boldsymbol{\rho}_{m}(\mathbf{k}+\mathbf{q})\right]+\rho(-\mathbf{k})\right. \\
& \times\left\{\mathbf{q} \cdot \mathbf{j}_{m}(\mathbf{k}+\mathbf{q}) k_{i}+\mathbf{k} \cdot \mathbf{q} j_{m i}(\mathbf{k}+\mathbf{q})\right\} \\
& \left.+\frac{1}{m} k_{i} \mathbf{k} \cdot \mathbf{q} \rho_{m}(\mathbf{q})\right]
\end{aligned}
$$

and the substitution of Eq. (33) into Eq. (25) yields

$$
\begin{aligned}
\dddot{j}_{\sigma}^{i}(\mathbf{q})= & \frac{1}{m V} \sum_{\mathbf{k}} v(\mathbf{k}) \\
& \times\left[i k_{i} \frac{\partial}{\partial t}\left[\rho(-\mathbf{k}) \rho_{\sigma}(\mathbf{k}+\mathbf{q})\right]+k_{i} \rho(-\mathbf{k}) \mathbf{q} \cdot \mathbf{j}_{\sigma}(\mathbf{k}+\mathbf{q})\right. \\
& \left.+\mathbf{k} \cdot \mathbf{q} j_{\sigma}^{i}(\mathbf{k}+\mathbf{q}) \rho(-\mathbf{k})\right] .
\end{aligned}
$$

Therefore we finally arrive at

$$
\begin{aligned}
\operatorname{Im} f_{\mathrm{xc}, \sigma \sigma^{\prime}}^{i j}(\mathbf{q}, \omega)= & -\frac{1}{V^{3} n_{\sigma} n_{\sigma^{\prime}}} \frac{1}{q^{2} \omega^{2}} \sum_{\mathbf{k}, \mathbf{k}^{\prime}} v(\mathbf{k}) v\left(\mathbf{k}^{\prime}\right) \operatorname{Im}\left[\omega^{2} k_{i} k_{j}^{\prime}\left\langle\left\langle\rho(-\mathbf{k}) \rho_{\sigma}(\mathbf{k}+\mathbf{q}) ; \rho\left(-\mathbf{k}^{\prime}\right) \rho_{\sigma^{\prime}}\left(\mathbf{k}^{\prime}-\mathbf{q}\right)\right\rangle\right\rangle\right. \\
& +\omega k_{j}^{\prime}\left(k_{i} q_{l}\left\langle\left\langle\rho(-\mathbf{k}) j_{\sigma}^{l}(\mathbf{k}+\mathbf{q}) ; \rho\left(-\mathbf{k}^{\prime}\right) \rho_{\sigma^{\prime}}\left(\mathbf{k}^{\prime}-\mathbf{q}\right)\right\rangle\right\rangle+\mathbf{k} \cdot \mathbf{q} \delta_{i l}\left\langle\left\langle j_{\sigma}^{l}(\mathbf{k}+\mathbf{q}) \rho(-\mathbf{k}) ; \rho\left(-\mathbf{k}^{\prime}\right) \rho_{\sigma^{\prime}}\left(\mathbf{k}^{\prime}-\mathbf{q}\right)\right\rangle\right\rangle\right) \\
& +\omega k_{i}\left(k_{j}^{\prime} q_{l}\left\langle\left\langle\rho(-\mathbf{k}) \rho_{\sigma}(\mathbf{k}+\mathbf{q}) ; \rho\left(-\mathbf{k}^{\prime}\right) j_{\sigma^{\prime}}^{l}\left(\mathbf{k}^{\prime}-\mathbf{q}\right)\right\rangle\right\rangle+\mathbf{k}^{\prime} \cdot \mathbf{q} \delta_{j l}\left\langle\left\langle\rho(-\mathbf{k}) \rho_{\sigma}(\mathbf{k}+\mathbf{q}) ; j_{\sigma^{\prime}}^{l}\left(\mathbf{k}^{\prime}-\mathbf{q}\right) \rho\left(-\mathbf{k}^{\prime}\right)\right\rangle\right\rangle\right) \\
& -\left\langle\left\langle k_{i} q_{l} \rho(-\mathbf{k}) j_{\sigma}^{l}(\mathbf{k}+\mathbf{q})+\mathbf{k} \cdot \mathbf{q} \delta_{i l} j_{\sigma}^{l}(\mathbf{k}+\mathbf{q}) \rho(-\mathbf{k}) ; k_{j}^{\prime} q_{l^{\prime}} \rho\left(-\mathbf{k}^{\prime}\right) j_{\sigma^{\prime}}^{l^{\prime}}\left(\mathbf{k}^{\prime}-\mathbf{q}\right)\right.\right. \\
& \left.\left.\left.+\mathbf{k}^{\prime} \cdot \mathbf{q} \delta_{j l^{\prime}} j_{\sigma^{\prime}}^{l^{\prime}}\left(\mathbf{k}^{\prime}-\mathbf{q}\right) \rho\left(-\mathbf{k}^{\prime}\right)\right\rangle\right\rangle\right] .
\end{aligned}
$$

In Eq. (35) and in the following formulas, a summation over the repeated indices $l$ and $l^{\prime}$ is understood.

Equation (35) is accurate to the order of $O\left(q^{0}\right)$. The singular term of $\operatorname{Im} f_{\mathrm{xc}, \sigma \sigma^{\prime}}^{L, T}(q, \omega)$ arises from the first term of the RHS of Eq. (35), which, to order $O\left(q^{-2}\right)$, yields

$$
\operatorname{Im} f_{\mathrm{xc}, \sigma \sigma^{\prime}}^{L, T}(q, \omega)=-\frac{1}{V^{3} n_{\sigma^{\prime}} n_{\sigma^{\prime}}} \frac{1}{q^{2}} \sum_{\mathbf{k}, \mathbf{k}^{\prime}} v(\mathbf{k}) v\left(\mathbf{k}^{\prime}\right) \frac{\mathbf{k} \cdot \mathbf{k}^{\prime}}{3} \operatorname{Im}\left\langle\left\langle\rho(-\mathbf{k}) \rho_{\sigma}(\mathbf{k}) ; \rho\left(-\mathbf{k}^{\prime}\right) \rho_{\sigma^{\prime}}\left(\mathbf{k}^{\prime}\right)\right\rangle\right\rangle .
$$

Based on simple symmetry considerations, the above equation can be rewritten as

$$
\begin{aligned}
\operatorname{Im} f_{\mathrm{xc}, \sigma \sigma^{\prime}}^{L, T}(q, \omega)= & \frac{\sigma \sigma^{\prime}}{V^{3} n_{\sigma^{\prime}} n_{\sigma^{\prime}}} \frac{1}{q^{2}} \sum_{\mathbf{k}, \mathbf{k}^{\prime}} v(\mathbf{k}) v\left(\mathbf{k}^{\prime}\right) \frac{\mathbf{k} \cdot \mathbf{k}^{\prime}}{3} \\
& \times \operatorname{Im}\left\langle\left\langle\rho_{\uparrow}(-\mathbf{k}) \boldsymbol{\rho}_{\downarrow}(\mathbf{k}) ; \boldsymbol{\rho}_{\uparrow}\left(\mathbf{k}^{\prime}\right) \boldsymbol{\rho}_{\downarrow}\left(-\mathbf{k}^{\prime}\right)\right\rangle\right\rangle .
\end{aligned}
$$

The structure of $f_{\mathrm{xc}, \sigma \sigma^{\prime}}^{L, T}(q, \omega)$ displayed in Eq. (7) is thus confirmed, and an explicit expression for $\operatorname{Im} A(\omega)$ is obtained:

$$
\begin{aligned}
\operatorname{Im} A(\omega)= & \frac{4}{3 V^{3} n^{2}} \sum_{\mathbf{k}, \mathbf{k}^{\prime}} v(\mathbf{k}) v\left(\mathbf{k}^{\prime}\right) \mathbf{k} \cdot \mathbf{k}^{\prime} \\
& \times \operatorname{Im}\left\langle\left\langle\rho_{\uparrow}(-\mathbf{k}) \rho_{\downarrow}(\mathbf{k}) ; \boldsymbol{\rho}_{\uparrow}\left(\mathbf{k}^{\prime}\right) \rho_{\downarrow}\left(-\mathbf{k}^{\prime}\right)\right\rangle\right\rangle .
\end{aligned}
$$

The results in this subsection hold for arbitrary spin polarization.

\section{B. High-frequency limit of $\operatorname{Im} A(\omega)$}

Because $\operatorname{Im} A(\omega)$ is antisymmetric with respect to $\omega$, we only need to consider positive $\omega$ for which 


$$
\begin{aligned}
\operatorname{Im}\left\langle\left\langle\rho_{\uparrow}(-\mathbf{k}) \rho_{\downarrow}(\mathbf{k}) ; \rho_{\uparrow}\left(\mathbf{k}^{\prime}\right) \rho_{\downarrow}\left(-\mathbf{k}^{\prime}\right)\right\rangle\right\rangle \\
=-\pi \sum_{n}\left\langle 0\left|\rho_{\uparrow}(-\mathbf{k}) \rho_{\downarrow}(\mathbf{k})\right| n\right\rangle\langle n| \rho_{\uparrow}\left(\mathbf{k}^{\prime}\right) \\
\quad \times \rho_{\downarrow}\left(-\mathbf{k}^{\prime}\right)|0\rangle \delta\left(\omega-\omega_{n 0}\right) .
\end{aligned}
$$

Here $\omega_{n 0}=E_{n}-E_{0}$ is an exact excitation energy of the system, with $E_{n}, E_{0}$ the excited and ground state energies, respectively: the sum extends to all the excited states that are coupled to the ground state by two density fluctuation operators.

At high frequency, the four-point response function should coincide with that of a noninteracting electron gas, which is given by

$$
\begin{gathered}
\operatorname{Im}\left\langle\left\langle\rho_{\uparrow}(-\mathbf{k}) \rho_{\downarrow}(\mathbf{k}) ; \rho_{\uparrow}\left(\mathbf{k}^{\prime}\right) \rho_{\downarrow}\left(-\mathbf{k}^{\prime}\right)\right\rangle\right\rangle^{(0)} \\
\quad \rightarrow \rightarrow \infty \\
\rightarrow-\pi \delta\left(\omega-k^{2} / m\right) \delta_{\mathbf{k}, \mathbf{k}^{\prime}} N_{\uparrow} N_{\downarrow}
\end{gathered}
$$

Therefore, from Eq. (38), one has

$\operatorname{Im} A(\omega) \stackrel{\omega \rightarrow \infty}{\rightarrow}-\frac{4 \pi n_{\uparrow} n_{\downarrow}}{3 V n^{2}} \sum_{\mathbf{k}} \delta\left(\omega-k^{2} / m\right) k^{2} v^{2}(k)$.

Carrying out the summation over $\mathbf{k}$ yields the result in Eq. (11), which holds for arbitrary spin polarization.

\section{High-frequency limit of $\operatorname{Im} B^{L, T}(\omega)$}

The high-frequency limit of $\operatorname{Im} f_{\mathrm{xc}}^{L}(\omega)$ was first established by Glick and Long. ${ }^{20}$ The extension to $\operatorname{Im} f_{\mathrm{xc}}^{T}(\omega)$ was carried out by Nifosi, Conti, and Tosi. ${ }^{13,14}$ In this subsection, we report the corresponding calculation for $\operatorname{Im} B^{L, T}(\omega)$. We limit ourselves to the paramagnetic case.

At high frequency, Eq. (35) reduces to

$$
\begin{aligned}
\operatorname{Im} f_{\mathrm{xc}, \sigma \sigma^{\prime}}^{i j}(q, \omega)= & \frac{1}{V^{3} n_{\sigma} n_{\sigma^{\prime}}} \frac{1}{q^{2} \omega^{2}} \sum_{\mathbf{k} \cdot \mathbf{k}^{\prime}} v(\mathbf{k}) v\left(\mathbf{k}^{\prime}\right) \operatorname{Im}\left[\omega^{2} k_{i} k_{j}^{\prime}\left\langle\left\langle\rho(-\mathbf{k}) \rho_{\sigma}(\mathbf{k}+\mathbf{q}) ; \rho\left(-\mathbf{k}^{\prime}\right) \rho_{\sigma^{\prime}}\left(\mathbf{k}^{\prime}-\mathbf{q}\right)\right\rangle\right\rangle+\omega k_{j}^{\prime}\left(k_{i} q_{l}+\mathbf{k} \cdot \mathbf{q} \delta_{i l}\right)\right. \\
& \times\left\langle\left\langle\rho(-\mathbf{k}) j_{\sigma}^{l}(\mathbf{k}+\mathbf{q}) ; \rho\left(-\mathbf{k}^{\prime}\right) \rho_{\sigma^{\prime}}\left(\mathbf{k}^{\prime}-\mathbf{q}\right)\right\rangle\right\rangle+\omega k_{i}\left(k_{j}^{\prime} q_{l}+\mathbf{k}^{\prime} \cdot \mathbf{q} \delta_{j l}\right)\left\langle\left\langle\rho(-\mathbf{k}) \rho_{\sigma}(\mathbf{k}+\mathbf{q}) ; \rho\left(-\mathbf{k}^{\prime}\right)\right.\right. \\
& \left.\left.\left.\times j_{\sigma^{\prime}}^{l}\left(\mathbf{k}^{\prime}-\mathbf{q}\right)\right\rangle\right\rangle+\left(k_{i} q_{l}+\mathbf{k} \cdot \mathbf{q} \delta_{i l}\right)\left(k_{j}^{\prime} q_{l^{\prime}}+\mathbf{k}^{\prime} \cdot \mathbf{q} \delta_{j l^{\prime}}\right)\left\langle\left\langle\rho(-\mathbf{k}) j_{\sigma}^{l}(\mathbf{k}+\mathbf{q}) ; \rho\left(-\mathbf{k}^{\prime}\right) j_{\sigma^{\prime}}^{l^{\prime}}\left(\mathbf{k}^{\prime}-\mathbf{q}\right)\right\rangle\right\rangle\right] . \quad(42)
\end{aligned}
$$

The last term in the above equation can be shown to make a contribution of order $\omega^{-5 / 2}$ to $\operatorname{Im} B^{L, T}(\omega)$, whereas the first three terms contribute to the leading order of $\omega^{-3 / 2}$.

We begin with the first term, which gives the leading order contribution to both $\operatorname{Im} A(\omega)$ and $\operatorname{Im} B^{L, T}(\omega)$. We denote it as the " $a$ " term in the derivations that follows. The four point response function can be rewritten as

$$
\begin{aligned}
\operatorname{Im}\left\langle\left\langle\rho(-\mathbf{k}) \rho_{\sigma}(\mathbf{k}+\mathbf{q}) ; \rho\left(-\mathbf{k}^{\prime}\right) \rho_{\sigma^{\prime}}\left(\mathbf{k}^{\prime}-\mathbf{q}\right)\right\rangle\right\rangle \\
=-\pi \sum_{\mathbf{p}_{1} \mathbf{p}_{2}} \sum_{\mathbf{p}_{3} \mathbf{p}_{4}} \sum_{\alpha \beta} \sum_{n} \delta\left(\omega-\epsilon_{\mathbf{p}_{1}-\mathbf{k}-\mathbf{q}}-\epsilon_{\mathbf{p}_{3}+\mathbf{k}}\right. \\
\left.\quad+\epsilon_{\mathbf{p}_{1}}+\epsilon_{\mathbf{p}_{3}}\right)\left\langle 0\left|a_{\mathbf{p}_{1} \sigma}^{+} a_{\mathbf{p}_{3} \alpha}^{+} a_{\mathbf{p}_{1}-\mathbf{k}-\mathbf{q} \sigma} a_{\mathbf{p}_{3}+\mathbf{k} \alpha}\right| n\right\rangle \\
\quad \times\left\langle n\left|a_{\mathbf{p}_{2}+\mathbf{k}^{\prime}-\mathbf{q} \sigma^{\prime}} a_{\mathbf{p}_{4}-\mathbf{k}^{\prime} \beta}^{+} a_{\mathbf{p}_{2} \sigma^{\prime}} a_{\mathbf{p}_{4} \beta}\right| 0\right\rangle,
\end{aligned}
$$

where $\epsilon_{\mathbf{p}_{1}}=p_{1}^{2} / 2 m$, etc. Obviously, $\left|\mathbf{p}_{1}\right|,\left|\mathbf{p}_{2}\right|,\left|\mathbf{p}_{3}\right|$, and $\left|\mathbf{p}_{4}\right|$ are all smaller than $k_{F}$. Therefore, to the leading order for large $\omega$, we have

$$
\begin{aligned}
\operatorname{Im}\left\langle\left\langle\rho(-\mathbf{k}) \rho_{\sigma}(\mathbf{k}+\mathbf{q}) ; \rho\left(-\mathbf{k}^{\prime}\right) \rho_{\sigma^{\prime}}\left(\mathbf{k}^{\prime}-\mathbf{q}\right)\right\rangle\right\rangle \\
\stackrel{\omega \rightarrow \infty}{\rightarrow}-\pi \sum_{\mathbf{p}_{1} \mathbf{p}_{2}} \sum_{\mathbf{p}_{3} \mathbf{p}_{4}} \sum_{\alpha \beta} \sum_{n} \delta\left(\omega-\epsilon_{\mathbf{k}+\mathbf{q}}-\epsilon_{\mathbf{k}}\right) \\
\quad \times\left\langle 0\left|a_{\mathbf{p}_{1} \sigma}^{+} a_{\mathbf{p}_{3} \alpha}^{+} a_{-\mathbf{k}-\mathbf{q} \sigma} a_{\mathbf{k} \alpha}\right| n\right\rangle \\
\quad \times\left\langle n\left|a_{\mathbf{k}^{\prime}-\mathbf{q} \sigma^{\prime}}^{+} a_{-\mathbf{k}^{\prime} \beta}^{+} a_{\mathbf{p}_{2} \sigma^{\prime}} a_{\mathbf{p}_{4} \beta}\right| 0\right\rangle .
\end{aligned}
$$

Carrying out the sum over the eigenstates of the noninteracting system (only double electron-hole pairs contribute), we get

$$
\begin{aligned}
\operatorname{Im}\left\langle\left\langle\rho(-\mathbf{k}) \rho_{\sigma}(\mathbf{k}+\mathbf{q}) ; \rho\left(-\mathbf{k}^{\prime}\right) \rho_{\sigma^{\prime}}\left(\mathbf{k}^{\prime}-\mathbf{q}\right)\right\rangle\right\rangle \\
\stackrel{\omega \rightarrow \infty}{\rightarrow}-\frac{\pi N^{2}}{4} \delta\left(\omega-k^{2} / m-q^{2} / 2 m-\mathbf{k} \cdot \mathbf{q} / m\right) \\
\quad \times\left\{\delta_{\mathbf{k}+\mathbf{q}, \mathbf{k}^{\prime}}+\delta_{\mathbf{k},-\mathbf{k}^{\prime}} \delta_{\sigma \sigma^{\prime}}-\delta_{\mathbf{k}+\mathbf{q}, \mathbf{k}^{\prime}} \delta_{\sigma \sigma^{\prime}}\right\}
\end{aligned}
$$

Substituting this result into Eq. (42), we come to

$$
\begin{aligned}
\operatorname{Im} f_{\mathrm{xc},-}^{i j, a}(\mathbf{q}, \omega)= & -\frac{\pi}{2 q^{4} V} \sum_{\mathbf{k}} \delta\left(\omega-k^{2} / m-q^{2} / 4 m\right) \\
& \times v(\mathbf{k}-\mathbf{q} / 2)\left(k_{i}-q_{i} / 2\right)[v(\mathbf{k}-\mathbf{q} / 2) \\
& \left.\times\left(k_{j}-q_{j} / 2\right)+v(\mathbf{k}+\mathbf{q} / 2)\left(k_{j}+q_{j} / 2\right)\right],
\end{aligned}
$$

where

$$
f_{\mathrm{xc},-}^{i j}(\mathbf{q}, \omega) \equiv \frac{1}{4} \sum_{\sigma \sigma^{\prime}} \sigma \sigma^{\prime} f_{\mathrm{xc}, \sigma \sigma^{\prime}}^{i j}(\mathbf{q}, \omega) .
$$

Therefore, 


$$
\begin{aligned}
\operatorname{Im} f_{\mathrm{xc},-}^{L, a}(q, \omega)= & -\frac{\pi}{2 q^{4} V} \sum_{\mathbf{k}} \delta\left(\omega-k^{2} / m-q^{2} / 4 m\right) \\
& \times v^{2}(k) \frac{1}{k^{4}}\left[2(\mathbf{k} \cdot \mathbf{q})^{2} k^{4}+4(\mathbf{k} \cdot \mathbf{q})^{4}\right. \\
& \left.-3(\mathbf{k} \cdot \mathbf{q})^{2} q^{2} k^{2}\right],
\end{aligned}
$$

where we have ignored terms that vanish for $q \rightarrow 0$. After carrying out the sum over $\mathbf{k}$ in Eq. (48), we obtain

$$
\operatorname{Im} f_{\mathrm{xc},-}^{L, a}(q, \omega)=-\frac{\pi e^{4}}{m^{1 / 2} \omega^{3 / 2}} \frac{1}{q^{2}}\left[\frac{4}{3} m \omega-\frac{7}{30} q^{2}\right] .
$$

For the transverse component, we need to evaluate the tensor product

$$
\begin{aligned}
\delta_{i j} \operatorname{Im} f_{\mathrm{xc},-}^{i j, a}(\mathbf{q}, \omega)= & -\frac{\pi}{2 q^{2} V} \sum_{\mathbf{k}} \delta\left(\omega-k^{2} / m-q^{2} / 4 m\right) \\
& \times v(\mathbf{k}-\mathbf{q} / 2)\left[v(\mathbf{k}-\mathbf{q} / 2)(\mathbf{k}-\mathbf{q} / 2)^{2}\right. \\
& \left.+v(\mathbf{k}+\mathbf{q} / 2)\left(k^{2}-q^{2} / 4\right)\right]
\end{aligned}
$$

or

$$
\begin{aligned}
\delta_{i j} \operatorname{Im} f_{\mathrm{xc},-}^{i j, a}(\mathbf{q}, \omega)= & -\frac{\pi}{2 q^{2} V} \sum_{\mathbf{k}} \delta\left(\omega-k^{2} / m-q^{2} / 4 m\right) v^{2}(k) \\
& \times\left[2 k^{2}-q^{2}+2(\mathbf{k} \cdot \mathbf{q})^{2} / k^{2}\right]
\end{aligned}
$$

to the order of $O\left(q^{0}\right)$. Equation (51) can be further simplified to

$$
\delta_{i j} \operatorname{Im} f_{\mathrm{xc},-}^{i j, a}(\mathbf{q}, \omega)=-\frac{\pi e^{4}}{m^{1 / 2} \omega^{3 / 2}} \frac{1}{q^{2}}\left[4 m \omega-\frac{q^{2}}{6}\right] .
$$

Thus, finally

$$
\operatorname{Im} f_{\mathrm{xc},-}^{T, a}(q, \omega)=-\frac{\pi e^{4}}{m^{1 / 2} \omega^{3 / 2}} \frac{1}{q^{2}}\left[\frac{4 m \omega}{3}+\frac{q^{2}}{30}\right] .
$$

Next we evaluate the contribution due to the second and third terms in Eq. (42). We denote them together as the " $b$ " term. At large $\omega$, the four point response functions are evaluated as

$$
\begin{aligned}
\operatorname{Im} & \left\langle\left\langle\rho(-\mathbf{k}) j_{\sigma}^{l}(\mathbf{k}+\mathbf{q}) ; \rho\left(-\mathbf{k}^{\prime}\right) \rho_{\sigma^{\prime}}\left(\mathbf{k}^{\prime}-\mathbf{q}\right)\right\rangle\right\rangle \\
= & -\frac{\pi N^{2}}{8 m}\left(k_{l}+q_{l}\right) \delta\left(\omega-k^{2} / m-q^{2} / 2 m-\mathbf{k} \cdot \mathbf{q} / m\right) \\
& \times\left\{\delta_{\mathbf{k}+\mathbf{q}, \mathbf{k}^{\prime}}+\delta_{\mathbf{k},-\mathbf{k}^{\prime}} \delta_{\sigma \sigma^{\prime}}-\delta_{\mathbf{k}+\mathbf{q}, \mathbf{k}^{\prime}} \delta_{\sigma \sigma^{\prime}}\right\}
\end{aligned}
$$

and

$$
\begin{aligned}
\operatorname{Im}\langle & \left.\left\langle\rho(-\mathbf{k}) \rho_{\sigma}(\mathbf{k}+\mathbf{q}) ; \rho\left(-\mathbf{k}^{\prime}\right) j_{\sigma^{\prime}}^{l}\left(\mathbf{k}^{\prime}-\mathbf{q}\right)\right\rangle\right\rangle \\
= & -\frac{\pi N^{2}}{8 m}\left(-k_{l}^{\prime}+q_{l}\right) \delta\left(\omega-k^{2} / m-q^{2} / 2 m-\mathbf{k} \cdot \mathbf{q} / m\right) \\
& \times\left\{\delta_{\mathbf{k}+\mathbf{q}, \mathbf{k}^{\prime}}+\delta_{\mathbf{k},-\mathbf{k}^{\prime}} \delta_{\sigma \sigma^{\prime}}-\delta_{\mathbf{k}+\mathbf{q}, \mathbf{k}^{\prime}} \delta_{\sigma \sigma^{\prime}}\right\}
\end{aligned}
$$

Substituting the above results into Eq. (42) leads to

$$
\begin{aligned}
\operatorname{Im} f_{\mathrm{xc},-}^{i j, b}(\mathbf{q}, \omega)= & \frac{\pi}{2 V m q^{2}} \frac{1}{\omega} \sum_{\mathbf{k}} \delta\left(\omega-k^{2} / m\right) v(\mathbf{k}-\mathbf{q} / 2) \mathbf{k} \cdot \mathbf{q} \\
& \times\left\{\left[\delta_{\sigma \bar{\sigma}^{\prime}} v(\mathbf{k}+\mathbf{q} / 2)+\delta_{\sigma \sigma^{\prime}} v(\mathbf{k}-\mathbf{q} / 2)\right]\right. \\
& \left.\times\left[k_{i} q_{j}+k_{j} q_{i}\right]-4 \delta_{\sigma \sigma^{\prime}} v(\mathbf{k}-\mathbf{q} / 2) k_{i} k_{j}\right\},
\end{aligned}
$$

where $\bar{\sigma}^{\prime}=-\sigma^{\prime}$. Therefore,

$$
\begin{aligned}
\operatorname{Im} f_{\mathrm{xc},-}^{i j, b}(\mathbf{q}, \omega)= & -\frac{2 \pi}{V m q^{2}} \frac{1}{\omega} \\
& \times \sum_{\mathbf{k}} v^{2}(\mathbf{k}) \delta\left(\omega-k^{2} / m\right) \frac{(\mathbf{k} \cdot \mathbf{q})^{2}}{k^{2}} k_{i} k_{j} .
\end{aligned}
$$

The longitudinal and transverse components can be further evaluated as

$$
\operatorname{Im} f_{\mathrm{xc},-}^{L, b}(\omega)=-\frac{8 \pi e^{4}}{5 m^{1 / 2} \omega^{3 / 2}}
$$

and

$$
\operatorname{Im} f_{\mathrm{xc},-}^{T, b}(\omega)=-\frac{8 \pi e^{4}}{15 m^{1 / 2} \omega^{3 / 2}}
$$

respectively.

Combining Eqs. (49), (53) and (58), (59) yields our complete results

$$
\operatorname{Im} f_{\mathrm{xc},-}^{L}(q, \omega)=-\frac{\pi e^{4}}{m^{1 / 2} \omega^{3 / 2}} \frac{1}{q^{2}}\left[\frac{4 m \omega}{3}+\frac{41 q^{2}}{30}\right]
$$

and

$$
\operatorname{Im} f_{\mathrm{xc},-}^{T}(q, \omega)=-\frac{\pi e^{4}}{m^{1 / 2} \omega^{3 / 2}} \frac{1}{q^{2}}\left[\frac{4 m \omega}{3}+\frac{17 q^{2}}{30}\right] .
$$

Thus we have the final results for $\operatorname{Im} B^{L, T}(\omega)$ as shown in Eq. (16). The singular term in the above equations is $\operatorname{Im} A(\omega)$, which is a special case of Eq. (11) for the paramagnetic state.

\section{High frequency limit of the real part xc kernel $\operatorname{Re} f_{\mathrm{xc}, \sigma \sigma^{\prime}}^{L, T}(q, \omega)$}

The third moment sum rule for $\operatorname{Re} f_{\mathrm{xc}, \sigma \sigma_{24}^{\prime}}^{L}(q, \infty)$ was first established by Goodman and Sjölander, ${ }^{24}$ and later rederived by Liu. ${ }^{25}$ Here we give derivations for both $\operatorname{Re} f_{\mathrm{xc}, \sigma \sigma^{\prime}}^{L}(q, \infty)$ and $\operatorname{Re} f_{\mathrm{xc}, \sigma \sigma^{\prime}}^{T}(q, \infty)$. We start from a rela- 
tion between $\operatorname{Re} f_{\mathrm{xc}, \sigma \sigma^{\prime}}^{\alpha}(q, \infty)$ and the first moment of the spin-current spin-current response function $M_{\sigma \sigma^{\prime}}^{\alpha}(\mathbf{q})$

$$
v^{\alpha}(q)+f_{\mathrm{xc}, \sigma \sigma^{\prime}}^{\alpha}(q, \infty)=\frac{m^{2}}{n_{\sigma} n_{\sigma^{\prime}}} \frac{M_{\sigma \sigma^{\prime}}^{\alpha}(\mathbf{q})-M_{\sigma \sigma^{\prime}}^{\alpha(0)}(\mathbf{q})}{q^{2}}
$$

where $\alpha=L, T$, and $v^{L}(q)=v(q)$, and $v^{T}(q)=0, M_{\sigma \sigma^{\prime}}^{\alpha(0)}(\mathbf{q})$ is the noninteracting version of $M_{\sigma \sigma^{\prime}}^{\alpha}(\mathbf{q})$. The first moment of the spin-current spin-current response function in Eq. (62) is defined as

$$
M_{\sigma \sigma^{\prime}}^{\alpha}(\mathbf{q})=-\frac{1}{\pi V} \int_{-\infty}^{\infty} d \omega \omega \operatorname{Im}\left\langle\left\langle j_{\sigma}^{\alpha}(\mathbf{q}, t), j_{\sigma^{\prime}}^{\alpha}(-\mathbf{q})\right\rangle\right\rangle
$$

which can also be rewritten as

$$
M_{\sigma \sigma^{\prime}}^{\alpha}(\mathbf{q})=\frac{i}{V}\left\langle\left[\dot{j}_{\sigma}^{\alpha}(\mathbf{q}), j_{\sigma^{\prime}}^{\alpha}(-\mathbf{q})\right]\right\rangle
$$

Note that the longitudinal component of $M_{\sigma \sigma^{\prime}}^{\alpha}(\mathbf{q})$ is related to the third moment of the spin-density spin-density response function via

$$
M_{\sigma \sigma^{\prime}}^{L}(q)=M_{3, \sigma \sigma^{\prime}}(q) / q^{2}
$$

By using Eq. (30), we have

$$
\begin{aligned}
& {\left[\dot{j}_{\sigma}^{i}(\mathbf{q}), j_{\sigma^{\prime}}^{j}(-\mathbf{q})\right]} \\
& \quad=\frac{i}{2 m}\left\{\left[\frac{2}{V} \sum_{\mathbf{k}} k_{i} \rho(-\mathbf{k}) \rho_{\sigma}(\mathbf{k}+\mathbf{q}) v(\mathbf{k}), j_{\sigma^{\prime}}^{j}(-\mathbf{q})\right]\right. \\
& \left.\quad-\frac{1}{m} \sum_{n} q_{l} Q_{n \sigma}\left[P_{n i} j_{n l}(\mathbf{q})+j_{n l}(\mathbf{q}) P_{n i}, j_{\sigma^{\prime}}^{j}(-\mathbf{q})\right]\right\} .
\end{aligned}
$$

The commutators on the RHS of the above equation can be carried out straightforwardly, though tediously, and one arrives at

$$
\begin{aligned}
{\left[\dot{j}_{\sigma}^{i}(\mathbf{q}), j_{\sigma^{\prime}}^{j}(-\mathbf{q})\right] } \\
=\frac{i}{m^{2} V} \sum_{\mathbf{k}} k_{i} v(\mathbf{k}) \rho\left[(-\mathbf{k})\left(k_{j}+q_{j}\right) \rho_{\sigma}(\mathbf{k}) \delta_{\sigma \sigma^{\prime}}\right. \\
\left.\quad-k_{j} \rho_{\sigma^{\prime}}(-\mathbf{k}-\mathbf{q}) \rho_{\sigma}(\mathbf{k}+\mathbf{q})\right] \\
\quad-\frac{i}{m^{3}} \sum_{n} \delta_{\sigma \sigma^{\prime}} Q_{n \sigma}\left[q_{i} \mathbf{q} \cdot \mathbf{P}_{n} P_{n j}+q_{j} P_{n i} \mathbf{q} \cdot \mathbf{P}_{n}\right. \\
\left.\quad+q^{2} P_{n i} P_{n j}\right] .
\end{aligned}
$$

We denote the contribution to $M_{\sigma \sigma^{\prime}}^{\alpha}(\mathbf{q})$ from the first term of the RHS of the above equation as $\left[M_{\sigma \sigma^{\prime}}^{\alpha}(\mathbf{q})\right]_{\text {int }}$ and that from the second term as $\left[M_{\sigma \sigma^{\prime}}^{\alpha}(\mathbf{q})\right]_{\text {kin }}$. They can be calculated as

$$
\begin{aligned}
{\left[M_{\sigma \sigma^{\prime}}^{\alpha}(\mathbf{q})\right]_{\mathrm{int}}=} & \frac{1}{m^{2}} q_{\alpha}^{2} v(q) n_{\sigma} n_{\sigma^{\prime}} \\
& +\frac{1}{m^{2}}\left[\delta_{\sigma \sigma^{\prime}} \sum_{\tau} \Gamma_{\sigma \tau}^{\alpha}(0)-\Gamma_{\sigma \sigma^{\prime}}^{\alpha}(\mathbf{q})\right]
\end{aligned}
$$

and

$$
\left[M_{\sigma \sigma^{\prime}}^{\alpha}(\mathbf{q})\right]_{\mathrm{kin}}=\frac{2}{3 V} \frac{1}{m^{2}} \delta_{\sigma \sigma^{\prime}}\left(2 q_{\alpha}^{2}+q^{2}\right)\left\langle T_{\sigma}\right\rangle,
$$

where

$$
\Gamma_{\sigma \sigma^{\prime}}^{\alpha}(\mathbf{q})=-\frac{1}{V^{2}} \sum_{\mathbf{k} \neq \mathbf{q}} k_{\alpha}^{2} v(\mathbf{k})\left\langle\rho_{\sigma}(\mathbf{q}-\mathbf{k}) \rho_{\sigma^{\prime}}(\mathbf{k}-\mathbf{q})\right\rangle,
$$

and $\left\langle T_{\sigma}\right\rangle$ is the spin-resolved kinetic energy. Substituting Eqs. (68), (69) into Eq. (62), one obtains

$$
\begin{aligned}
f_{\mathrm{xc}, \sigma \sigma^{\prime}}^{L, T}(\infty)= & \frac{1}{n_{\sigma^{\prime}}}\left[\alpha^{L, T} \delta_{\sigma \sigma^{\prime}} t_{c \sigma}+\frac{1}{q^{2} n_{\sigma}}\right. \\
& \left.\times\left\{\delta_{\sigma \sigma^{\prime}} \sum_{\tau} \Gamma_{\sigma \tau}^{\alpha}(0)-\Gamma_{\sigma \sigma^{\prime}}^{\alpha}(\mathbf{q})\right\}\right] .
\end{aligned}
$$

At small wave vector, it can be shown that

$$
\begin{aligned}
\Gamma_{\sigma \sigma^{\prime}}^{\alpha}(\mathbf{q})= & -\frac{4 \pi e^{2} n_{\sigma} n_{\sigma^{\prime}}}{3}\left[g_{\sigma \sigma^{\prime}}(0)-1\right] \\
& -\frac{1}{2} \beta^{\alpha} n_{\sigma} n_{\sigma^{\prime}} q^{2} \int d \mathbf{r} v(r)\left[g_{\sigma \sigma^{\prime}}(r)-1\right]
\end{aligned}
$$

to the accuracy of $O\left(q^{2}\right)$. After some straightforward algebra, one obtains the following result: ${ }^{7}$

$$
\begin{aligned}
f_{\mathrm{xc}, \sigma \sigma^{\prime}}^{L, T}(q, \infty) \stackrel{q \rightarrow 0}{\rightarrow} & -\frac{4 \pi e^{2}}{3 q^{2}} \frac{n_{\uparrow} n_{\downarrow}}{n_{\sigma} n_{\sigma^{\prime}}}\left[g_{\uparrow \downarrow}(0)-1\right] \sigma \sigma^{\prime} \\
& +\alpha^{L, T} \frac{t_{c \sigma}}{n_{\sigma}} \delta_{\sigma \sigma^{\prime}}+\frac{1}{2} \beta^{L, T} \\
& \times \int d \mathbf{r} \frac{e^{2}}{r}\left[g_{\sigma \sigma^{\prime}}(r)-1\right] .
\end{aligned}
$$

The results in this subsection are valid for arbitrary spin polarization.

\section{E. Proof of $\operatorname{Re} A(0)=0$}

The vanishing of $\operatorname{Re} A(0)$ follows from the fact that $(1 / \pi) \int_{-\infty}^{\infty}[\operatorname{Im} A(\omega) / \omega] d \omega$ is equal to (minus) the first moment of the spin-current spin-current response function in the spin channel, which, by gauge invariance and the continuity equation, coincides with the third moment of the spin channel of the spin-density spin-density response function, i.e., $-A(\infty)$. More explicitly, from Eq. (7), one has 


$$
\frac{1}{\pi} \int_{-\infty}^{\infty} \frac{\operatorname{Im} A(\omega)}{\omega} d \omega=\frac{1}{\pi} \lim _{q \rightarrow 0} \int_{-\infty}^{\infty} d \omega \frac{q^{2}}{\omega} \operatorname{Im} f_{\mathrm{xc},-}^{L}(q, \omega) .
$$

The above equation can be rewritten, making use of Eq. (24), as

$$
\begin{aligned}
\frac{1}{\pi} \int_{-\infty}^{\infty} \frac{\operatorname{Im} A(\omega)}{\omega} d \omega= & \frac{m^{2}}{\pi N n} \lim _{q \rightarrow 0} \int_{-\infty}^{\infty} d \omega \omega \\
& \times \sum_{\sigma \sigma^{\prime}} \sigma \sigma^{\prime} \operatorname{Im}\left\langle\left\langle j_{\sigma}^{L}(\mathbf{q}, t) ; j_{\sigma^{\prime}}^{L}(-\mathbf{q})\right\rangle\right\rangle .
\end{aligned}
$$

As can be seen from Eq. (63), the RHS of the above equation is $-(m / n)^{2} \Sigma_{\sigma \sigma^{\prime}} \sigma \sigma^{\prime} M_{\sigma \sigma^{\prime}}^{L}(q)$, which coincides, for small $q$, with $-A(\infty)$ according to Eqs. (65) and (12). Thus, $A(0)$ $=0$, and this conclusion holds for arbitrary spin polarization.

\section{F. Low frequency limit of $\operatorname{Re} B^{L, T}(\omega)$}

The low frequency limit of the xc kernel for the density channel in the case of a paramagnetic state was first related to the Landau parameters in Ref. 21. In this subsection, we extend the results of Ref. 21 to the spin channel.

The quasiclassical quasiparticle Hamiltonian is

$$
\begin{aligned}
H_{\mathrm{qp}}(\mathbf{r}, \mathbf{p}, \sigma)= & \boldsymbol{\epsilon}_{\mathbf{p}+\mathbf{A}_{\sigma^{\prime}}(\mathbf{r}, t), \sigma}+\sum_{\mathbf{p}^{\prime} \sigma^{\prime}} f_{\mathbf{p} \sigma, \mathbf{p}^{\prime} \sigma^{\prime}} \\
& \times\left[n_{\mathbf{p}^{\prime} \sigma^{\prime}}-n_{0}\left(\epsilon_{\mathbf{p}^{\prime}+\mathbf{A}_{\sigma^{\prime}}(\mathbf{r}, t), \sigma^{\prime}}\right)\right],
\end{aligned}
$$

where $\epsilon_{\mathbf{p} \sigma}$ is the energy of a quasiparticle of spin $\sigma$, $\nabla_{\mathbf{p}} \epsilon_{\mathbf{p} \sigma} \equiv \mathbf{v}_{\mathbf{p} \sigma}=\mathbf{p} / m_{\sigma}^{*}$ is the quasiparticle velocity, $m_{\sigma}^{*}$ is the effective mass, and $f_{\mathbf{p} \sigma, \mathbf{p}^{\prime} \sigma^{\prime}}$ are the Landau interaction functions. Here $\mathbf{A}_{\sigma}(\mathbf{r}, t)$ is a spin-resolved vector potential that couples only to $\sigma$-spin particles. We assume that $\mathbf{A}_{\sigma}(\mathbf{r}, t)$ is small. Linearizing with respect to $\mathbf{A}_{\sigma}(\mathbf{r}, t)$ we get

$$
\begin{aligned}
H_{\mathrm{qp}}(\mathbf{r}, \mathbf{p}, \sigma)= & \boldsymbol{\epsilon}_{\mathbf{p} \sigma^{+}}+\sum_{\mathbf{p}^{\prime} \sigma^{\prime}} f_{\mathbf{p} \sigma, \mathbf{p}^{\prime} \sigma^{\prime}} n_{1 \mathbf{p}^{\prime} \sigma^{\prime}} \\
& +\sum_{\sigma^{\prime}} \mathbf{A}_{\sigma^{\prime}} \cdot\left[\mathbf{v}_{\mathbf{p} \sigma^{\prime}} \delta_{\sigma \sigma^{\prime}}\right. \\
& \left.-\sum_{\mathbf{p}^{\prime}} \mathbf{v}_{\mathbf{p}^{\prime} \sigma^{\prime}} n_{0}^{\prime}\left(\epsilon_{\mathbf{p}^{\prime} \sigma}\right) f_{\mathbf{p} \sigma, \mathbf{p}^{\prime} \sigma^{\prime}}\right],
\end{aligned}
$$

where $n_{1 \mathbf{p} \sigma}(\mathbf{r}, t)=n_{\mathbf{p} \sigma}(\mathbf{r}, t)-n_{0}\left(\epsilon_{\mathbf{p} \sigma}\right)$. The Liouville equation of motion for the quasiparticle distribution function is governed by the above Hamiltonian. After linearization and Fourier transformation with respect to space and time we obtain

$$
\begin{aligned}
& \left(\mathbf{q} \cdot \mathbf{v}_{\mathbf{p} \sigma}-\omega\right) n_{1 \mathbf{p} \sigma}(\mathbf{q}, \omega)-\mathbf{q} \cdot \mathbf{v}_{\mathbf{p} \sigma} n_{0}^{\prime}\left(\epsilon_{\mathbf{p} \sigma}\right) \\
& \times\left[\sum_{\sigma^{\prime}} \mathbf{A}_{\sigma^{\prime}}(\mathbf{q}, \omega) \cdot\left\{\mathbf{v}_{\mathbf{p} \sigma^{\prime}} \delta_{\sigma \sigma^{\prime}}-\sum_{\mathbf{p}^{\prime}} \boldsymbol{\nabla}_{\mathbf{p}^{\prime}} n_{\mathbf{p}^{\prime} \sigma^{\prime}} f_{\mathbf{p} \sigma, \mathbf{p}^{\prime} \sigma^{\prime}}\right\}\right. \\
& \left.\quad+\sum_{\mathbf{p}^{\prime} \sigma^{\prime}} f_{\mathbf{p} \sigma, \mathbf{p}^{\prime} \sigma^{\prime}} n_{1 \mathbf{p}^{\prime} \sigma^{\prime}}(\mathbf{q}, \omega)\right]=0
\end{aligned}
$$

In a paramagnetic system this equation reduces to

$$
\begin{aligned}
& \left(\mathbf{q} \cdot \mathbf{v}_{\mathbf{p}}-\omega\right) n_{1 \mathbf{p} \sigma}(\mathbf{q}, \omega)-\mathbf{q} \cdot \mathbf{v}_{\mathbf{p}} n_{0}^{\prime}\left(\boldsymbol{\epsilon}_{\mathbf{p}}\right) \\
& \quad \times\left[\sum_{\sigma^{\prime}} \mathbf{A}_{\sigma^{\prime}}(\mathbf{q}, \omega) \cdot\left\{\mathbf{v}_{\mathbf{p}} \delta_{\sigma \sigma^{\prime}}-\sum_{\mathbf{p}^{\prime}} \nabla_{\mathbf{p}^{\prime}} n_{\mathbf{p}^{\prime}} f_{\mathbf{p} \sigma, \mathbf{p}^{\prime} \sigma^{\prime}}\right\}\right. \\
& \left.\quad+\sum_{\mathbf{p}^{\prime} \sigma^{\prime}} f_{\mathbf{p} \sigma, \mathbf{p}^{\prime} \sigma^{\prime}} n_{1 \mathbf{p}^{\prime} \sigma^{\prime}}(\mathbf{q}, \omega)\right]=0
\end{aligned}
$$

which can be further simplified to

$$
\begin{aligned}
& \left(\mathbf{q} \cdot \mathbf{v}_{\mathbf{p}}-\omega\right) n_{1 \mathbf{p}}^{a}(\mathbf{q}, \omega) \\
& \quad-2 \mathbf{q} \cdot \mathbf{v}_{\mathbf{p}} n_{0}^{\prime}\left(\epsilon_{\mathbf{p}}\right)\left[\sum_{\mathbf{p}^{\prime}} f_{\mathbf{p} \cdot \mathbf{p}^{\prime}}^{a} n_{1 \mathbf{p}^{\prime}}^{a}(\mathbf{q}, \omega)\right. \\
& \left.\quad+\frac{1}{m_{s}} \mathbf{p} \cdot \mathbf{A}_{a}(\mathbf{q}, \omega)\right]=0,
\end{aligned}
$$

where $n_{1 \mathbf{p}}^{a}=n_{1 \mathbf{p} \uparrow}-n_{1 \mathbf{p} \downarrow}$, and $f_{\mathbf{p} \cdot \mathbf{p}^{\prime}}^{a}$ is the spin-antisymmetric component of the Landau interaction function. In writing Eq. (80), we have introduced two new objects, namely, the spin mass $m_{s}$,

$$
\frac{1}{m_{s}}=\frac{1}{m^{*}}\left[1+\frac{1}{3} F_{1}^{a}\right],
$$

and the "spin-channel vector potential"

$$
\mathbf{A}_{a}(\mathbf{q}, \omega)=\frac{1}{2}\left[\mathbf{A}_{\uparrow}(\mathbf{q}, \omega)-\mathbf{A}_{\downarrow}(\mathbf{q}, \omega)\right] .
$$

The physical significance of the spin mass is discussed extensively in Ref. 27. In brief, it turns out that the spin current, defined as the difference between spin-resolved currents

$$
\mathbf{j}_{a}(\mathbf{q}, \omega)=\mathbf{j}_{\uparrow}(\mathbf{q}, \omega)-\mathbf{j}_{\downarrow}(\mathbf{q}, \omega),
$$

is related to the quasiparticle distribution function in the following manner:

$$
\mathbf{j}_{a}(\mathbf{q}, \omega)=\sum_{\mathbf{p}} \frac{\mathbf{p}}{m_{s}} n_{1 \mathbf{p}}^{a}(\mathbf{q}, \omega)+\frac{n}{m_{s}} \mathbf{A}_{a}(\mathbf{q}, \omega) .
$$

The response of $\mathbf{j}_{a}(\mathbf{q}, \omega)$ due to the perturbation $\mathbf{A}_{a}(\mathbf{q}, \omega)$ is given by

$$
j_{a}^{L, T}(q, \omega)=\chi_{s}^{L, T}(q, \omega) A_{a}^{L, T}(q, \omega) .
$$

Combining Eqs. (80) and (84), we can compute the small- $q$ limit of the response function with the following results: 


$$
\chi_{s}^{L}(q, \omega)-\frac{n}{m_{s}} \rightarrow \frac{n q^{2} k_{F}^{2}}{m_{s}^{2} m^{*} \omega^{2}}\left[\frac{3}{5}+\frac{4 F_{2}^{a}}{75}+\frac{F_{0}^{a}}{3}\right]
$$

and

$$
\chi_{s}^{T}(q, \omega)-\frac{n}{m_{s}} \rightarrow \frac{n q^{2} k_{F}^{2}}{m_{s}^{2} m^{*} \omega^{2}}\left[\frac{1}{5}+\frac{F_{2}^{a}}{25}\right] .
$$

On the other hand, we also have

$$
\chi_{s}^{L, T}(q, \omega)=\frac{\chi_{0}^{L, T}(q, \omega)}{1-B^{L, T}(q, \omega) \chi_{0}^{L, T}(q, \omega) q^{2} / \omega^{2}},
$$

which implies

$$
\chi_{0}^{L, T}(q, \omega) \stackrel{q \rightarrow 0}{\rightarrow} \frac{n}{m}\left[1+\gamma^{L, T} \frac{E_{F} q^{2}}{m \omega^{2}}\right],
$$

where $\gamma_{L}=6 / 5$ and $\gamma_{T}=2 / 5$. Comparing the $O\left(q^{0}\right)$ term of $\chi_{s}^{L, T}(q, \omega)$ in Eqs. (86), (87) with the corresponding term in Eq. (89), we find

$$
\frac{m_{s}}{m}=1-\frac{n A(\omega)}{m \omega^{2}} .
$$

Substituting Eq. (90) back into Eq. (88) yields

$$
\chi_{s}^{L, T}(q, \omega) \stackrel{q \rightarrow 0}{\rightarrow} \frac{n}{m_{s}}\left[1+\gamma_{L, T} \frac{\epsilon_{F} q^{2}}{m_{s} \omega^{2}}+\frac{n q^{2}}{m_{s} \omega^{2}} B^{L, T}(\omega)\right]
$$

Comparison of Eq. (91) with Eqs. (86) and (87), respectively, leads to Eqs. (18) and (19), and to the further relation

$$
B^{L}(0)-\frac{4}{3} B^{T}(0)=\left.\frac{1}{n} \frac{\partial^{2}}{\partial \zeta^{2}} \epsilon_{\mathrm{xc}}(\zeta)\right|_{\zeta=0},
$$

where $\epsilon_{\mathrm{xc}}(\zeta)$ is the xc energy for per particle at spin polarization $\zeta$. Notice that to obtain Eq. (92) we have used the relation

$$
\left.\frac{\partial^{2}}{\partial \zeta^{2}} \epsilon_{\mathrm{xc}}(\zeta)\right|_{\zeta=0}=\frac{2 E_{F}}{3} \frac{F_{0}^{a}-F_{1}^{s} / 3}{1+F_{1}^{s} / 3},
$$

which can be deduced from the well known relation between the spin susceptibility and the Landau parameters $F_{1}^{s}$ and $F_{1}^{a}$ in Fermi liquid theory. ${ }^{26}$ Equation (92) combined with the corresponding relation for the density channel ${ }^{28}$ yields

$$
\lim _{\omega \rightarrow 0} \frac{f_{\mathrm{xc}}^{L}(\omega)-4 f_{\mathrm{xc}}^{T}(\omega) / 3-\partial^{2}\left(n \epsilon_{\mathrm{xc}}\right) / \partial n^{2}}{\omega}=0,
$$

thus confirming the exact identity

$$
\lim _{\omega \rightarrow 0} \frac{f_{\sigma \sigma^{\prime}}^{L}(\omega)-4 f_{\sigma \sigma^{\prime}}^{T}(\omega) / 3-\partial^{2}\left(n \epsilon_{\mathrm{xc}}\right) / \partial n_{\sigma} \partial n_{\sigma^{\prime}}}{\omega}=0
$$

which was first reported in Ref. 7.

\section{G. Low frequency limit of $\operatorname{Im} A(\omega)$}

Equation (15) - the low frequency limit of $\operatorname{Im} A(\omega)$-is established within the mode-decoupling approximation, which is exact only in the high density limit. The analytic form of this approximation is given by Eq. (14) of Ref. 7, which, in the paramagnetic case, reduces to

$$
\begin{aligned}
\operatorname{Im} A(\omega)= & -\frac{4}{3 n^{2} V} \sum_{\mathbf{q}} v_{\mathbf{q}}^{2} q^{2} \\
& \times \int_{0}^{\omega} \frac{d \omega^{\prime}}{\pi} \frac{\operatorname{Im} \chi_{0 \uparrow}\left(q, \omega-\omega^{\prime}\right) \operatorname{Im} \chi_{0 \uparrow}\left(q, \omega^{\prime}\right)}{\left|\epsilon\left(q, \omega-\omega^{\prime}\right)\right|^{2}\left|\epsilon\left(q, \omega^{\prime}\right)\right|^{2}} \\
& \times\left\{\left[1+\Theta\left(q, \omega-\omega^{\prime}\right)\right]\left[1+\Theta\left(q, \omega^{\prime}\right)\right]\right. \\
& \left.-\Theta\left(q, \omega-\omega^{\prime}\right) \Theta\left(q, \omega^{\prime}\right)\right\},
\end{aligned}
$$

where $\Theta(q, \omega)=2 v(q)\left[-\operatorname{Re} \chi_{0 \uparrow}(q, \omega)+v(q)\left|\chi_{0 \uparrow}(q, \omega)\right|^{2}\right]$. To leading order in $\omega$, for small $\omega$, Eq. (96) simplifies to

$$
\begin{aligned}
\operatorname{Im} A(\omega)= & -\frac{4}{3 n^{2} V} \sum_{\mathbf{q}} v_{\mathbf{q}}^{2} q^{2} \frac{1}{|\epsilon(q, 0)|^{2}} \\
& \times \int_{0}^{\omega} \frac{d \omega^{\prime}}{\pi} \operatorname{Im} \chi_{0 \uparrow}\left(q, \omega-\omega^{\prime}\right) \operatorname{Im} \chi_{0 \uparrow}\left(q, \omega^{\prime}\right),
\end{aligned}
$$

where $\chi_{0 \uparrow}(q, \omega)$ is the Lindhard function for just one spin component. At small frequency the limiting form $\operatorname{Im} \chi_{0 \uparrow}(q, \omega) \simeq m^{2} \omega / 4 \pi q$ for $q \leqslant 2 k_{F}$ can be applied, and thus we get

$$
\begin{aligned}
\operatorname{Im} A(\omega) \rightarrow & -\frac{1}{3 n^{2} V}\left(\frac{m^{2}}{2 \pi}\right)^{2} \frac{1}{6 \pi} \omega^{3} \sum_{\mathbf{q}} v(q)^{2} \\
& \times \frac{1}{|\epsilon(q, 0)|^{2}} \theta\left(2 k_{F}-q\right) .
\end{aligned}
$$

Making use of the RPA for the static dielectric function, one obtains, after some straightforward calculations, the result of Eq. (15).

\section{H. Low frequency limit of $\operatorname{Im} B^{L, T}(\omega)$}

Again, we restrict our attention to the paramagnetic case in this subsection. At small $\omega$, Eq. (35) reduces to

$\operatorname{Im} f_{\mathrm{xc}, \sigma \sigma^{\prime}}^{i j}(\mathbf{q}, \omega)$

$$
\begin{aligned}
= & -\frac{1}{V^{3} n_{\sigma^{\prime}} n_{\sigma^{\prime}}} \frac{1}{q^{2} \omega^{2}} \sum_{\mathbf{k}, \mathbf{k}^{\prime}} v(\mathbf{k}) v\left(\mathbf{k}^{\prime}\right) \\
& \times\left(k_{i} q_{l}+\mathbf{k} \cdot \mathbf{q} \delta_{i l}\right)\left(k_{j}^{\prime} q_{l^{\prime}}+\mathbf{k}^{\prime} \cdot \mathbf{q} \delta_{j l^{\prime}}\right) \\
& \times\left\langle\left\langle\rho(-\mathbf{k}) j_{\sigma}^{l}(\mathbf{k}+\mathbf{q}) ; \rho\left(-\mathbf{k}^{\prime}\right) j_{\sigma^{\prime}}^{l}\left(\mathbf{k}^{\prime}-\mathbf{q}\right)\right\rangle\right\rangle .
\end{aligned}
$$


As discussed in our previous analysis of the density channel, ${ }^{12}$ two types of processes contribute to the four-point correlation function $\left\langle\left\langle\rho(-\mathbf{k}) j_{\sigma}^{l}(\mathbf{k}+\mathbf{q}) ; \rho\left(-\mathbf{k}^{\prime}\right) j_{\sigma^{\prime}}^{l^{\prime}}\left(\mathbf{k}^{\prime}\right.\right.\right.$ $-\mathbf{q})\rangle\rangle$ in this limit: direct processes, and exchange processes. We first evaluate the contribution of direct processes [denoted by $(D)$ below], for which

$$
\begin{aligned}
\operatorname{Im}\langle\langle A B ; C D\rangle\rangle_{\omega}= & -\frac{1}{\pi} \int_{0}^{\omega} d \omega^{\prime}\left[\operatorname{Im}\langle\langle A ; C\rangle\rangle_{\omega^{\prime}}\right. \\
& \times \operatorname{Im}\langle\langle B ; D\rangle\rangle_{\omega-\omega^{\prime}} \\
& \left.+\operatorname{Im}\langle\langle A ; D\rangle\rangle_{\omega^{\prime}} \operatorname{Im}\langle\langle B ; C\rangle\rangle_{\omega-\omega^{\prime}}\right] .
\end{aligned}
$$

Their contribution is therefore

$$
\begin{aligned}
\operatorname{Im} f_{\mathrm{xc}, \sigma \sigma^{\prime}}^{i j(D)}(\mathbf{q}, \omega)= & \frac{1}{V^{3} n_{\sigma} n_{\sigma^{\prime}}} \frac{1}{q^{2} \omega^{2}} \sum_{\mathbf{k}, \mathbf{k}^{\prime}} v(\mathbf{k}) v\left(\mathbf{k}^{\prime}\right)\left(k_{i} q_{l}+\mathbf{k} \cdot \mathbf{q} \delta_{i l}\right)\left(k_{j}^{\prime} q_{l^{\prime}}+\mathbf{k}^{\prime} \cdot \mathbf{q} \delta_{j l^{\prime}}\right) \int_{0}^{\omega} \frac{d \omega^{\prime}}{\pi}\left[\operatorname{Im} \chi\left(-\mathbf{k}, \omega-\omega^{\prime}\right)\right. \\
& \left.\times \operatorname{Im} \chi_{\sigma \sigma^{\prime}}^{l l^{\prime}}\left(\mathbf{k}, \omega^{\prime}\right) \delta_{\mathbf{k},-\mathbf{k}^{\prime}}-\frac{k^{l} k^{l^{\prime}}}{k^{4}} \omega^{\prime}\left(\omega-\omega^{\prime}\right) \operatorname{Im} \chi_{\sigma \sigma}\left(\mathbf{k}, \omega^{\prime}\right) \operatorname{Im} \chi_{\sigma^{\prime} \sigma^{\prime}}\left(-\mathbf{k}, \omega-\omega^{\prime}\right) \delta_{\mathbf{k}, \mathbf{k}^{\prime}}\right],
\end{aligned}
$$

where $\chi\left(-\mathbf{k}, \omega^{\prime}\right)$, etc., are proper linear response functions. In a paramagnetic system, the longitudinal and transverse components of the xc kernel can be obtained as

$$
\begin{aligned}
\operatorname{Im} f_{\mathrm{xc}, \sigma \sigma^{\prime}}^{L, T(D)}(\omega)= & -\frac{8}{5 N^{2} \omega^{2}} \sum_{\mathbf{k}} v^{2}(\mathbf{k}) \int_{0}^{\omega} \frac{d \omega^{\prime}}{\pi}\left[k^{2} \operatorname{Im} \chi\left(-\mathbf{k}, \omega-\omega^{\prime}\right)\left\{\lambda_{1}^{L, T} \operatorname{Im} \chi_{\sigma \sigma^{\prime}}^{L}\left(\mathbf{k}, \omega^{\prime}\right)+\lambda_{2}^{L, T} \operatorname{Im} \chi_{\sigma \sigma^{\prime}}^{T}\left(\mathbf{k}, \omega^{\prime}\right)\right\}\right. \\
& \left.+\lambda_{3}^{L, T} \operatorname{Im} \chi_{\sigma \sigma}\left(\mathbf{k}, \omega^{\prime}\right) \operatorname{Im} \chi_{\sigma^{\prime} \sigma^{\prime}}\left(-\mathbf{k}, \omega-\omega^{\prime}\right) \omega^{\prime}\left(\omega-\omega^{\prime}\right)\right],
\end{aligned}
$$

where $\lambda_{1}^{L}=2, \quad \lambda_{1}^{T}=2 / 3, \quad \lambda_{2}^{L}=4 / 3, \quad \lambda_{2}^{T}=1, \quad \lambda_{3}^{L}=2$, and $\lambda_{3}^{T}$ $=2 / 3$. The leading order contribution at small $\omega$ is

$$
\begin{aligned}
\operatorname{Im} B^{L, T(D)}(\omega)= & -\frac{2}{5 N^{2}} \lambda_{2}^{L, T} \frac{1}{\omega^{2}} \sum_{\mathbf{k}} v^{2}(\mathbf{k}) \\
& \times \int_{0}^{\omega} \frac{d \omega^{\prime}}{\pi} \operatorname{Im} \chi\left(-\mathbf{k}, \omega-\omega^{\prime}\right) \\
& \times \operatorname{Im} \chi_{s}^{T}\left(\mathbf{k}, \omega^{\prime}\right) k^{2} .
\end{aligned}
$$

We note that, in RPA

$$
\chi^{T}(k, \omega)=\chi_{s}^{T}(k, \omega)=\chi_{0}^{T}(k, \omega)
$$

and

$$
\chi^{L}(k, \omega)=\frac{\chi_{0}^{L}(k, \omega)}{\epsilon(k, \omega)},
$$

where $\epsilon(k, \omega)=1-v(k) \chi_{0}(k, \omega)$ is the RPA dielectric function. Therefore, ${ }^{12}$

$$
\operatorname{Im} B^{L, T(D)}(\omega)=\operatorname{Im} f_{\mathrm{xc}}^{L, T(D)}(\omega) .
$$

It should be evident from the above argument that Eq. (106) holds only within the RPA.

Under the same RPA for the screened Coulomb potential one can show that exchange processes only contribute to the spin-diagonal terms $f_{\mathrm{xc}, \sigma \sigma}$ and therefore make equivalent contributions to the density and the spin channel [see Eqs. (8) and (9)]. We conclude that

$$
\operatorname{Im} B^{L, T}(\omega)=\operatorname{Im} f_{\mathrm{xc}}^{L, T}(\omega) .
$$

The low frequency limit of $\operatorname{Im} f_{\mathrm{xc}}^{L, T}(\omega)$ is given by Eq. (15) in Ref. 12. Combining that result with Eq. (107) leads to the expression of Eq. (20).

\section{INTERPOLATION FORMULAS}

We now present our interpolation formula for $\operatorname{Im} B^{L, T}(\omega)$ in paramagnetic state, which incorporates all the exact results listed in Sec. II,

$$
\begin{aligned}
\operatorname{Im} B^{L, T}(\omega)= & -\frac{2 \omega_{\mathrm{p} 1}}{n} \tilde{\omega}\left[\frac{a^{L, T}}{\left(1+b^{L, T} \widetilde{\omega}^{2}\right)^{5 / 4}}\right. \\
& \left.+\widetilde{\omega}^{2} e^{-\left(|\tilde{\omega}|-\Omega^{L, T}\right)^{2} / \Gamma^{L, T}}\right],
\end{aligned}
$$

where $\tilde{\omega}=\omega / \omega_{m}$, with $\omega_{m}$ the position of the "collective peak" discussed below. The form of the interpolation is completely analogous to the one we recently proposed for the density channel. ${ }^{12}$ However, there is a difference in the value of $\omega_{m}$ between the density channel and the spin channel. In the density channel, $\omega_{m}=2 \omega_{\mathrm{pl}}$, where $\omega_{\mathrm{pl}}$ is the plasmon frequency. But, in the spin channel case, a simple estimate of the position of the peak, based on the mode-decoupling approximation, suggests $\omega_{m} \simeq 3 \omega_{\mathrm{pl}} / 2$ (see Appendix A).

Requiring that Eq. (108) has a peak at $\omega_{m}$ yields the relation

$$
\Omega^{L, T}=1-\frac{3 \Gamma^{L, T}}{2} .
$$

The low frequency limit of Eq. (20) fixes $a^{L, T}$ as 
TABLE I. The parameters for $B^{L}(\omega)$ [Eq. (108)].

\begin{tabular}{lcccc}
\hline \hline$r_{s}$ & $10^{2} a^{L}$ & $b^{L}$ & $\Gamma^{L}$ & $\Omega^{L}$ \\
\hline 1 & 0.3769 & 0.2145 & 1.831 & -1.747 \\
3 & 0.8191 & 0.2065 & 1.526 & -1.289 \\
5 & 1.069 & 0.1881 & 1.393 & -1.090 \\
\hline \hline
\end{tabular}

$$
a^{L, T}=\frac{1}{\pi \alpha} r_{s}^{2} S^{L, T}
$$

where $\alpha=(4 / 9 \pi)^{1 / 3}$. The high frequency limit of Eq. (16) fixes $b^{L, T}$ as

$$
b^{L, T}=16\left(\frac{4}{3}\right)^{7 / 5} r_{s}-3 / 5\left[\frac{a^{L, T}}{c^{L, T}}\right]^{4 / 5} .
$$

Finally, from Eq. (10), we have the sum rule

$$
\begin{aligned}
& -\left(\frac{4 \sqrt{2 \pi} a^{L, T}}{[\Gamma(1 / 4)]^{2} \sqrt{b^{L, T}}}+\frac{1}{2 \pi}\left\{2 \Omega^{L, T} \Gamma^{L, T} e^{-\left(\Omega^{L, T}\right)^{2} / \Gamma^{L, T}}\right.\right. \\
& \left.\left.+\left(\pi \Gamma^{L, T}\right)^{1 / 2}\left[\Gamma^{L, T}+2\left(\Omega^{L, T}\right)^{2}\right]\left[1+\operatorname{erf}\left(\frac{\Omega^{L, T}}{\sqrt{\Gamma^{L, T}}}\right)\right]\right\}\right) \\
& \quad \times \frac{2 \omega_{\mathrm{pl}}}{n}=B^{L, T}(0)-B^{L, T}(\infty),
\end{aligned}
$$

where

$$
\operatorname{erf}(x)=\frac{2}{\sqrt{\pi}} \int_{0}^{x} e^{-y^{2}} d y
$$

$\Gamma^{L, T}$ and $\Omega^{L, T}$ are further determined from Eqs. (109) and (112). This completes the determination of the parameters appearing in Eq. (108).

Next we present some numerical results at typical densities. Before doing this, however, we must choose input values for $B^{L, T}(0)$ and $B^{L, T}(\infty)$. We use the Landau parameters calculated by Yasuhara and Ousaka ${ }^{29}$ to determine $B^{L, T}(0)$ via Eqs. (18) and (19). On the other hand, $B^{L, T}(\infty)$ is calculated from Eq. (17). We make use of the approximate correlation energy $\epsilon_{c}$ proposed by Vosko, Wilk, and Nusair, ${ }^{30}$ which is based on the results of Monte Carlo calculation, ${ }^{31}$ to calculate $t_{c}$ via the relation

$$
t_{c}=-\epsilon_{c}+3 n \frac{d \epsilon_{c}}{d n}
$$

TABLE II. The parameters for $B^{T}(\omega)$ [Eq. (108)].

\begin{tabular}{ccccc}
\hline \hline$r_{s}$ & $10^{2} a^{T}$ & $b^{T}$ & $\Gamma^{T}$ & $\Omega^{T}$ \\
\hline 1 & 0.2827 & 0.3447 & 1.935 & -1.903 \\
3 & 0.6143 & 0.3318 & 1.511 & -1.267 \\
5 & 0.8020 & 0.3022 & 1.375 & -1.063 \\
\hline \hline
\end{tabular}

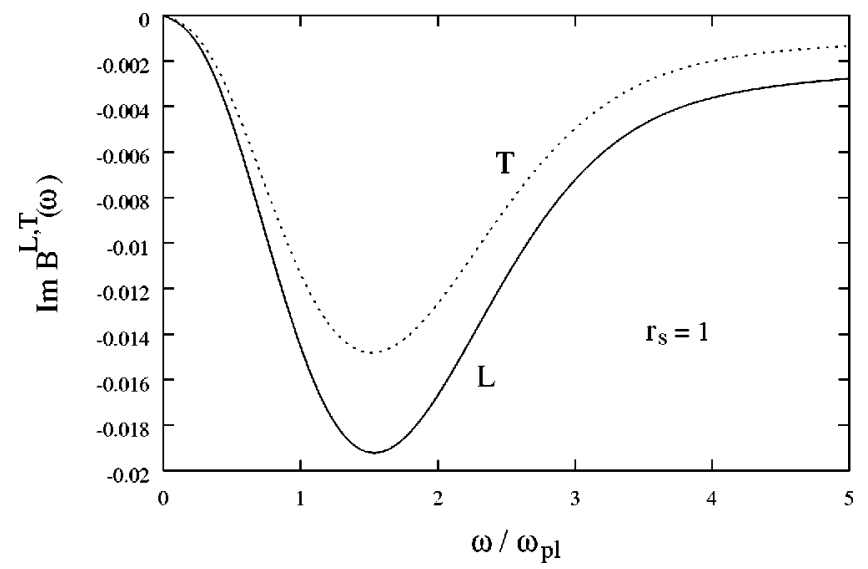

FIG. 1. Imaginary part of $B^{L}(\omega)$ and $B^{T}(\omega)$ at $r_{s}=1$ in units of $2 \omega_{\mathrm{pl}} / n$, as functions of $\omega$.

For the spin-resolved pair correlation function $g_{\sigma \sigma^{\prime}}(r)$, we use the values obtained by diffusion Monte Carlo (Table VIII) in Ref. 32.

The values of the parameters of Eq. (108) are presented for three values of the Wigner-Seitz radius $r_{s}=1,3$, and 5 in Table I. We note that, although $\operatorname{Im} B^{L, T}(\omega) \simeq \operatorname{Im} f_{\text {xc }}^{L, T}(\omega)$ at low frequency, the parameters $a^{L, T}$ differ from the corresponding ones in the density channel (reported in our Table II and Table III of Ref. 12), because of the difference between $\omega_{m}$ and $2 \omega_{\mathrm{pl}}$. Plots of $\operatorname{Im} B^{L, T}(\omega)$ and $\operatorname{Re} B^{L, T}(\omega)$ are presented in Figs. 1-6.

Given the exact results for $A(\omega)$ listed in Sec. II, it would be natural to use for $\operatorname{Im} A(\omega)$ the same form of interpolation that we are proposing for $\operatorname{Im} B^{L, T}(\omega)$. Unfortunately this can only be done at very small $r_{s}$. At $r_{s}>1$, the low-frequency and high-frequency limits are such that the integral $(1 / \pi) \int_{0}^{\infty}[\operatorname{Im} A(\omega) / \omega] d \omega$ calculated from this type of interpolation always exceeds $-A(\infty)$. For this reason we are forced to take a different approach, already described in Ref. 7: we first calculate $\operatorname{Im} A(\omega)$ in the mode-decoupling approximation, ${ }^{13,14}$ and then correct the low-frequency behavior by a frequency-dependent factor $g(\omega),{ }^{7}$ chosen in such way that the exact high frequency behavior is maintained and the sum rule

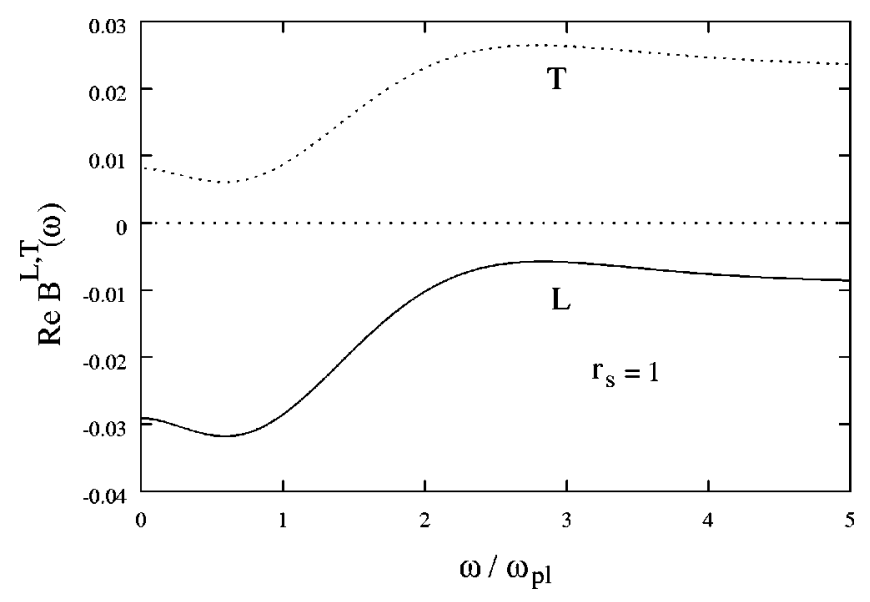

FIG. 2. Real part of $B^{L}(\omega)$ and $B^{T}(\omega)$ at $r_{s}=1$. Notations and units are as in Fig. 1. 


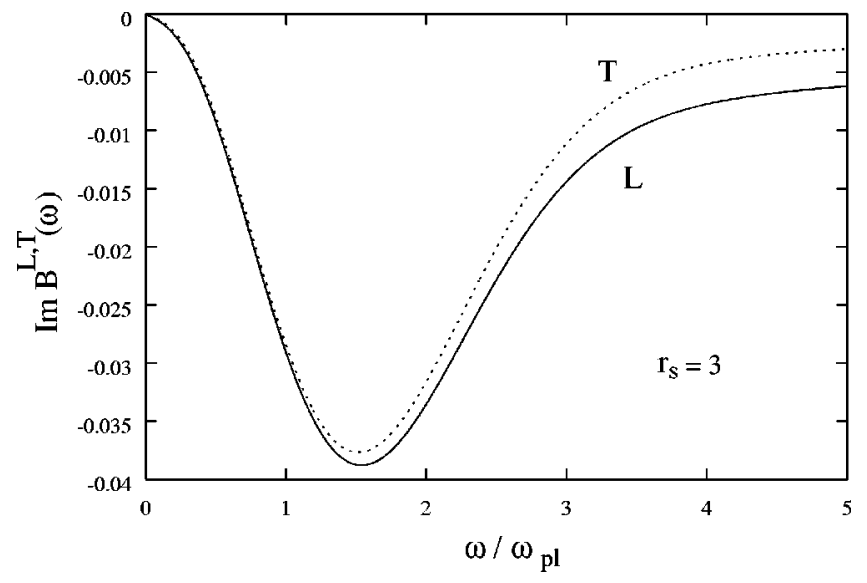

FIG. 3. Imaginary part of $B^{L}(\omega)$ and $B^{T}(\omega)$ at $r_{s}=3$. Notations and units are as in Fig. 1.

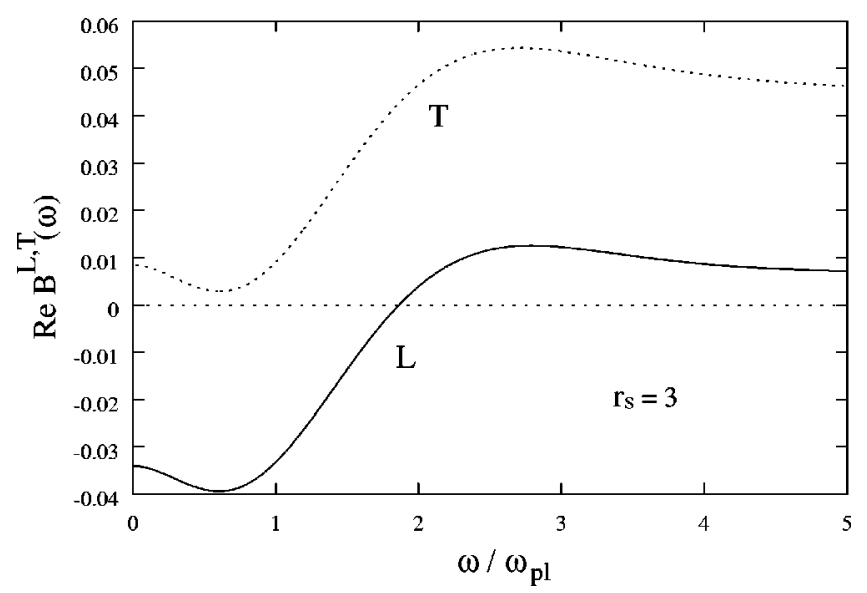

FIG. 4. Real part of $B^{L}(\omega)$ and $B^{T}(\omega)$ at $r_{s}=3$. Notations and units are as in Fig. 1.

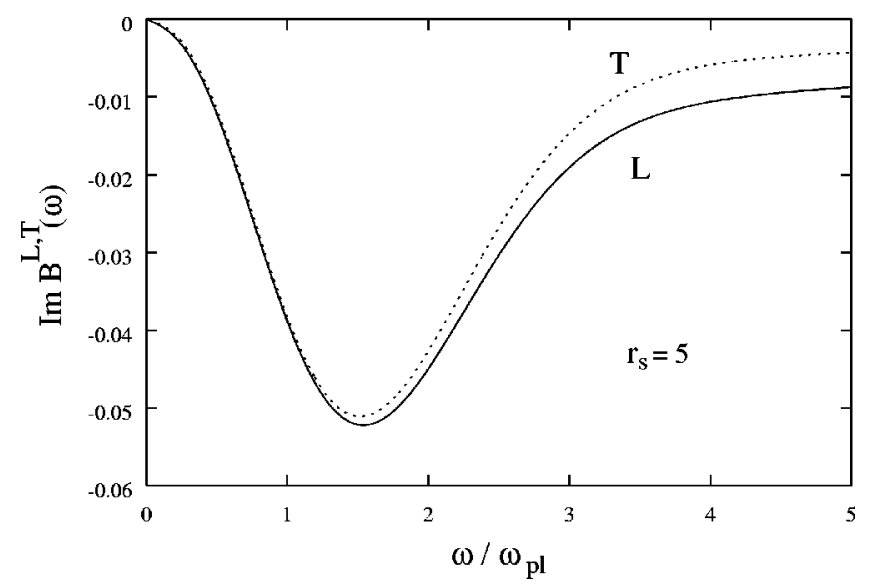

FIG. 5. Imaginary part of $B^{L}(\omega)$ and $B^{T}(\omega)$ at $r_{s}=5$. Notations and units are as in Fig. 1.

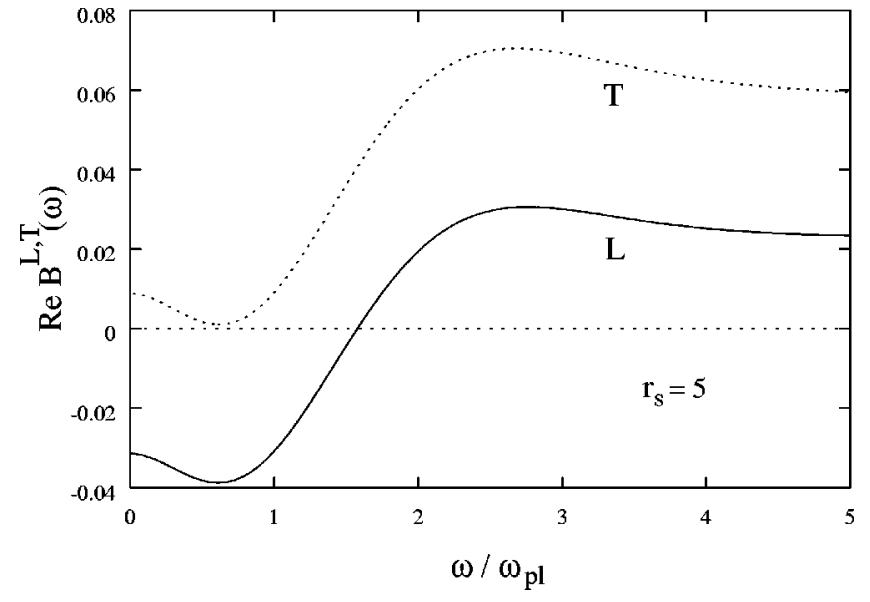

FIG. 6. Real part of $B^{L}(\omega)$ and $B^{T}(\omega)$ at $r_{s}=5$. Notations and units are as in Fig. 1.

$$
\operatorname{Re} A(0)-\operatorname{Re} A(\infty)=P \int_{-\infty}^{\infty} \frac{d \omega^{\prime}}{\pi} \frac{\operatorname{Im} A\left(\omega^{\prime}\right)}{\omega^{\prime}}
$$

is satisfied. This approach has the merit of producing a peak at approximately the same frequency as the mode-decoupling approximation, which we feel is physically justified.

\section{SUMMARY}

Several exact results for the singular and regular components $A(\omega)$ and $B^{L, T}(\omega)$, respectively, of the exchangecorrelation kernel of an electron liquid in the spin channel have been obtained. Based on these results, we have proposed an interpolation formula for $\operatorname{Im} B^{L, T}(\omega)$ in the paramagnetic state at any frequency $\omega$.

The results obtained in this paper constitute progress in the study of the many-body local field factors in the electron liquid. These and the proposed interpolation formulas will be useful in applications of the time dependent spin density function theory.

\section{ACKNOWLEDGMENTS}

We gratefully acknowledge support for this work from the NSF Grant No. DMR-0074959 and from the Research Board Grant No. URB-00-029 at the University of Missouri.

\section{APPENDIX A: POSITION OF THE COLLECTIVE PEAK OF $\operatorname{Im} B^{L, T}(\omega)$}

This appendix is devoted to explaining why the position of the peak in $\operatorname{Im} B^{L, T}(\omega)$ vs frequency is at about $3 \omega_{\mathrm{pl}} /$ 2 (in paramagnetic case) and not at $2 \omega_{\mathrm{pl}}$ as in the density channel. To this end, we denote the $O\left(q^{0}\right)$ term of $f_{\sigma \sigma^{\prime}}^{i j}(\mathbf{q}, \omega)$ as $B_{\sigma \sigma^{\prime}}^{i j}(\mathbf{q}, \omega)$, and define $B^{i j}(\mathbf{q}, \omega)$ $=\Sigma_{\sigma \sigma^{\prime}}\left(n_{\sigma^{\prime}} n_{\sigma^{\prime}} / n^{2}\right) B_{\sigma \sigma^{\prime}}^{i j}(\mathbf{q}, \omega)$.

In the RPA-based mode-decoupling approximation the imaginary part of the $\mathrm{xc}$ kernel is given by 


$$
\begin{aligned}
\operatorname{Im} B^{i j}(\omega)= & -\frac{1}{V N^{2} \omega^{2}} \frac{1}{\pi} \lim _{q \rightarrow 0} \frac{1}{q^{2}} \int_{0}^{\omega} d \omega^{\prime} \sum_{\mathbf{k}} v^{2}(\mathbf{k})\left[\omega\left\{\omega k_{i} k_{j}+\left(\omega-\omega^{\prime}\right) \frac{1}{(\mathbf{k}+\mathbf{q})^{2}}\left[2 k_{i} k_{j}(2 \mathbf{k}+\mathbf{q}) \cdot \mathbf{q}+\mathbf{k} \cdot \mathbf{q}\left(k_{j} q_{i}+k_{i} q_{j}\right)\right]\right\}\right. \\
& \times \operatorname{Im} \chi^{(\mathrm{RPA})}\left(-\mathbf{k}, \omega^{\prime}\right) \operatorname{Im} \chi_{s}^{(\mathrm{RPA})}\left(\mathbf{k}+\mathbf{q}, \omega-\omega^{\prime}\right)+\left(k_{i} q_{l}+\mathbf{k} \cdot \mathbf{q} \delta_{i l}\right)\left(k_{j} q_{l^{\prime}}+\mathbf{k} \cdot \mathbf{q} \delta_{j l^{\prime}}\right) \operatorname{Im} \chi^{(\mathrm{RPA})}\left(-\mathbf{k}, \omega^{\prime}\right) \\
& \left.\times \operatorname{Im} \chi_{s}^{l l^{\prime}(\mathrm{RPA})}\left(\mathbf{k}+\mathbf{q}, \omega-\omega^{\prime}\right)\right] .
\end{aligned}
$$

In the plasmon pole approximation $\operatorname{Im} \chi^{(\mathrm{RPA})}\left(-\mathbf{k}, \omega^{\prime}\right)$ is proportional to $\delta\left[\omega^{\prime}-\Omega_{\mathrm{pl}}(k)\right]$ where $\Omega_{\mathrm{pl}}(k)$ is the dispersion of the plasmon. Noticing that $\omega \geqslant \omega^{\prime}$ in Eq. (A1) and recalling that $\Omega_{\mathrm{pl}}(k) \geqslant \omega_{\mathrm{pl}}$, we see that $\operatorname{Im} B^{i j}(\omega)$ can be different from zero only for

$$
\omega \geqslant \omega_{\mathrm{pl}} \text {. }
$$

As we pointed out in Sec. III H, in RPA one has

$$
\chi_{s}^{L, T}(q, \omega)=\chi_{0}^{L, T}(q, \omega) .
$$

Therefore $\operatorname{Im} \chi_{s}^{l l^{\prime}(\mathrm{RPA})}\left(\mathbf{k}+\mathbf{q}, \omega-\omega^{\prime}\right)$ differs from zero $\chi_{s}^{L, T}\left(\mathbf{k}+\mathbf{q}, \omega-\omega^{\prime}\right)$ only when the condition

$$
\omega-\omega^{\prime}<\left[k_{F}|\mathbf{k}+\mathbf{q}|+|\mathbf{k}+\mathbf{q}|^{2} / 2\right] / m
$$

is satisfied. Going to the $q \rightarrow 0$ limit, and using the fact that $\omega^{\prime}=\Omega_{\mathrm{pl}}(k)$, we can recast the above condition in the form

$$
\omega<\Omega_{\mathrm{pl}}(k)+\frac{1}{m}\left[k_{F} k+k^{2} / 2\right] .
$$

In order to get a rough estimate of the value of $\omega$ beyond which the plasmon contribution to $\operatorname{Im} B^{i j}(\omega)$ drops to zero we now restrict the sum over $\mathbf{k}$ to wave vectors that satisfy the condition $k<k_{c}$, where $k_{c}$ is the wave vector at which the plasmon enters the electron-hole continuum [i.e., $\left.\omega_{\mathrm{pl}}\left(k_{c}\right)=k_{F} k_{c} / m+k_{c}^{2} / 2 m\right]$. Assuming also that the plasmon is nearly dispersionless, i.e., that $\Omega_{\mathrm{pl}}(k) \simeq \omega_{\mathrm{pl}}$ for $k \leqslant k_{c}$, we see that Eq. (A5) becomes

$$
\omega<2 \omega_{\mathrm{pl}} .
$$

Combining Eqs. (A2) and (A6) we see that the collective contribution to $\operatorname{Im} B^{i j}(\omega)$ is significant only in the range $\omega_{\mathrm{pl}} \leqslant \omega<2 \omega_{\mathrm{pl}}$, and therefore its peak value can be expected to occur at about midrange, i.e., at $\omega=3 \omega_{\mathrm{pl}} / 2$.

\section{APPENDIX B: PROOF OF Eq. (23)}

In Ref. 12 we proved that in a paramagnetic electron system the low frequency limits of the longitudinal and trans- verse kernels in the density channel are related as follows:

$$
\lim _{\omega \rightarrow 0} \frac{\operatorname{Im} f_{\mathrm{xc}}^{T}(\omega)}{\omega}=\frac{3}{4} \lim _{\omega \rightarrow 0} \frac{\operatorname{Im} f_{\mathrm{xc}}^{L}(\omega)}{\omega} .
$$

We now show that this relation also holds in the spin channel. The proof is completely similar to the one given in Ref. 12 for the density channel and depends on the fact that

$$
\begin{aligned}
\operatorname{Im} B_{\sigma \sigma^{\prime}}^{i j}(\mathbf{q}, \omega)= & -\frac{4}{N^{2} V \omega^{2}} \frac{1}{V^{3}} \lim \frac{1}{q \rightarrow 0} q^{2} \\
& \times \sum_{\mathbf{k}, \mathbf{k}^{\prime}} \operatorname{Im}\left\langle\left\langle j_{\sigma}^{l}(\mathbf{k}) \rho(-\mathbf{k}) ; j_{\sigma^{\prime}}^{l^{\prime}}\left(\mathbf{k}^{\prime}\right)\right.\right. \\
& \left.\left.\times \rho\left(-\mathbf{k}^{\prime}\right)\right\rangle\right\rangle v(\mathbf{k}) v\left(\mathbf{k}^{\prime}\right) T_{i j l l^{\prime}}\left(\mathbf{k}, \mathbf{k}^{\prime}, \mathbf{q}\right),
\end{aligned}
$$

where

$$
T_{i j l l^{\prime}}\left(\mathbf{k}, \mathbf{k}^{\prime}, \mathbf{q}\right)=\left(k_{i} q_{l}+\delta_{i l} \mathbf{q} \cdot \mathbf{k}\right)\left(k_{j}^{\prime} q_{l^{\prime}}+\delta_{j l} \mathbf{k}^{\prime} \cdot \mathbf{q}\right) .
$$

Here again the summation over the repeated indices $l$ and $l^{\prime}$ is assumed. It was shown in Ref. 12 that, to leading order in $\omega$,

$$
\begin{aligned}
& \int \Omega_{\mathbf{q}} \sum_{i j} \delta_{i j} q^{2} T_{i j l l^{\prime}}\left(\mathbf{k}, \mathbf{k}^{\prime}, \mathbf{q}\right) \\
& =\frac{5}{2} \int d \Omega_{\mathbf{q}} \sum_{i j} q_{i} q_{j} T_{i j l l^{\prime}}\left(\mathbf{k}, \mathbf{k}^{\prime}, \mathbf{q}\right) .
\end{aligned}
$$

The key point is that this identity does not depend on spin indices. Therefore its substitution into Eq. (B2) yields

$$
\sum_{i j} \delta_{i j} \operatorname{Im} B_{\sigma \sigma^{\prime}}^{i j}(\mathbf{q}, \omega) / q^{2}=\frac{5}{2} \sum_{i j} \operatorname{Im} B_{\sigma \sigma^{\prime}}^{i j}(\mathbf{q}, \omega) q_{i} q_{j} / q^{4},
$$

which implies Eq. (23).

\footnotetext{
${ }^{1}$ K.S. Singwi and M.P. Tosi, in Solid State Physics, edited by H. Ehrenreich, F. Seitz, and D. Turnbull (Academic, New York, 1981), Vol. 36, p. 177.

${ }^{2}$ S. Ichimaru, Rev. Mod. Phys. 54, 1017 (1982).
}

\footnotetext{
${ }^{3}$ P. Hohenberg and W. Kohn, Phys. Rev. 136, B864 (1964).

${ }^{4}$ W. Kohn and L.J. Sham, Phys. Rev. 140, A1133 (1965).

${ }^{5}$ E. Runge and E.K.U. Gross, Phys. Rev. Lett. 77, 2037 (1984).

${ }^{6}$ E.K.U. Gross, J.F. Dobson, and M. Petersilka, in Topics in
} 
Current Chemistry, edited by R.F. Nalewajski (Springer, Berlin, 1996).

${ }^{7}$ Z. Qian, A. Constantinescu, and G. Vignale, Phys. Rev. Lett. 90, 066402 (2003)

${ }^{8}$ C.A. Kukkonen and A.W. Overhauser, Phys. Rev. B 20, 550 (1979).

${ }^{9}$ G. Vignale and K.S. Singwi, Phys. Rev. B 32, 2156 (1985).

${ }^{10}$ C.F. Richardson and N.W. Ashcroft, Phys. Rev. B 50, 8170 (1994); G.S. Atwal, I.G. Khalil, and N.W. Ashcroft, ibid. 67, 115107 (2003).

${ }^{11}$ C.A. Kukkonen and J.W. Wilkins, Phys. Rev. B 19, 6075 (1979).

${ }^{12}$ Z. Qian and G. Vignale, Phys. Rev. B 65, 235121 (2002).

${ }^{13}$ S. Conti, R. Nifos, and M.P. Tosi, J. Phys.: Condens. Matter 9, L475 (1997).

${ }^{14}$ R. Nifos, S. Conti, and M.P. Tosi, Phys. Rev. B 58, 12758 (1998).

${ }^{15}$ G. Vignale and W. Kohn, Phys. Rev. Lett. 77, 2037 (1996).

${ }^{16} \mathrm{G}$. Vignale and W. Kohn, in Electronic Density Functional Theory, edited by J. Dobson, M.P. Das, and G. Vignale (Plenum Press, New York, 1996).

${ }^{17}$ G. Vignale, C.A. Ullrich, and S. Conti, Phys. Rev. Lett. 79, 4878 (1997).
${ }^{18}$ P.L. de Boeij, F. Kootstra, J.A. Berger, R. van Leeuwen, and J.G. Snijders, J. Chem. Phys. 115, 1995 (2001).

${ }^{19}$ M. van Faassen, P.L. de Boeij, R. van Leeuwen, J.A. Berger, and J.G. Snijders, Phys. Rev. Lett. 88, 186401 (2002).

${ }^{20}$ A.J. Glick and W.F. Long, Phys. Rev. B 4, 3455 (1971).

${ }^{21}$ S. Conti and G. Vignale, Phys. Rev. B 60, 7966 (1999).

${ }^{22}$ E.K.U. Gross and W. Kohn, Phys. Rev. Lett. 55, 2850 (1985); 57, 923(E) (1986).

${ }^{23}$ N. Iwamoto and E.K.U. Gross, Phys. Rev. B 35, 3003 (1987).

${ }^{24}$ B. Goodman and A. Sjölander, Phys. Rev. B 8, 200 (1973).

${ }^{25}$ K.L. Liu, Can. J. Phys. 69, 573 (1991).

${ }^{26}$ D. Pines and P. Nozières, The Theory of Quantum Liquids (Benjamin, New York, 1966), Vol. 1.

${ }^{27}$ Z. Qian, G. Vignale, and D. C. Marinescu (unpublished).

${ }^{28}$ C.A. Ullrich and G. Vignale, Phys. Rev. B 65, 245102 (2002).

${ }^{29}$ H. Yasuhara and Y. Ousaka, Int. J. Mod. Phys. B 6, 3089 (1992).

${ }^{30}$ S.H. Vosko, L. Wilk, and M. Nusair, Can. J. Phys. 58, 1200 (1980).

${ }^{31}$ D.M. Ceperley and B.J. Alder, Phys. Rev. Lett. 45, 566 (1980).

${ }^{32}$ G. Ortiz and P. Ballone, Phys. Rev. B 50, 1391 (1994). 